\title{
Condition-based prediction of time-dependent reliability in composites
}

\author{
Juan Chiachío $^{\mathrm{a}, *}$, Manuel Chiachío ${ }^{\mathrm{a}}$, Shankar Sankararaman $^{\mathrm{b}}$, Abhinav Saxena $^{\mathrm{b}}$, Kai Goebel $^{\mathrm{c}}$ \\ ${ }^{a}$ Dept. Structural Mechanics and Hydraulic Engineering, University of Granada, Spain \\ ${ }^{b}$ SGT Inc., NASA Ames Research Center, Moffett Field, CA 94035-1000 \\ ${ }^{c}$ NASA Ames Research Center, Intelligent Systems Division. Moffett Field, CA 94035-1000
}

\begin{abstract}
This paper presents a reliability-based prediction methodology to obtain the remaining useful life of composite materials subjected to fatigue degradation. Degradation phenomena such as stiffness reduction and increase in matrix micro-cracks density are sequentially estimated through a Bayesian filtering framework that incorporates information from both, multi-scale damage models and damage measurements, that are sequentially collected along the process. A set of damage states are further propagated forward in time by simulating the damage progression using the models in absence of new damage measurements to estimate the time-dependent reliability of the composite material. As a key contribution, the estimation of the remaining useful life is obtained as a probability from the prediction of the time-dependent reliability, whose validity is formally proven using the axioms of Probability Logic. A case study is presented using multi-scale fatigue damage data from a cross-ply carbon-epoxy laminate.
\end{abstract}

Keywords: Model-based prognostics, Time-dependent reliability, Fatigue, Composites.

\section{Introduction}

In general, the problem of damage prognosis is challenging [1-3] not only due to its complexity and multidisciplinary nature, but also for its direct impact on safety and cost. While structural health monitoring (SHM) technology has experienced a considerable development over the past two decades, little effort has gone into integrating SHM with prognostics science for lifecycle reassessment and condition-based maintenance [4]. The latter is especially significant for composite materials due to their increasing use in high-performance applications such as aeronautics or space. Composites are well-known for their high strength-to-weight ratios, but also for being susceptible to damage from the beginning of lifespan $[5,6]$. This damage can be hard to detect [7] and usually becomes a critical issue for reliability and competitiveness

\footnotetext{
*Corresponding author. e-mail: jchiachio@ugr.es Tel:(+34)958240037 Fax:(+34)958249959

E.T.S. de Ingenieros de Caminos, Canales y Puertos. Campus de Fuentenueva s/n, 18071, Granada
} 
of composite structures [8]. Continuous assessment of the health state using state-of-the-art SHM technology, and based on that, the prediction of the remaining time until which the structure is expected to continue performing the required function, is of key importance for the efficient and reliable use of composite materials.

Damage prognostics can be defined as the estimation of the remaining useful life (RUL) of a system based on knowledge about the current damage state and the future degradation process of the system [1]. The methodology for damage prognostics typically consists of two main steps: first, an estimation of the current damage state based on (incomplete) up-to-date information from the system response given by SHM data; and second, a propagation forward in time of the updated state estimate (while no new SHM data are available) until the failure threshold is reached. The inherent complexity of this process implies uncertainty that comes not only from uncertain system inputs (upcoming loads, environmental conditions, etc.) but also from the lack of knowledge about the physics of the damage process. This uncertainty can increase dramatically when dealing with full-scale composite structures in real environments. Thus, probability-based frameworks are best suited for prognostics, rather than deterministic or point-valued RUL estimations [9].

In the literature, there is a growing number of articles dealing with probability-based approaches for damage prognostics, for example in the areas of rotating machinery [10-12], pneumatic valves [13, 14], fatigue of metals [15-17], just to name but a few. Depending on the chosen modeling option for forward damage propagation, approaches can be roughly classified into model-based or data-driven [18]. However, in general, the number of contributions in the context of composites is still very limited [19-21], where the benefits of the probability-based prognostics approach can be fully exploited to deal with the variability and complexity of the degradation process in composites.

In the present paper, a model-based prognostics framework is proposed in application to fatigue degradation in composite materials. For the problem of damage state estimation, which takes place before the problem of damage prognostics, an approach based on particle filters $(\mathrm{PF})[22,23]$ is adopted to sequentially estimate the joint probability density function (PDF) of damage states and model parameters as long as new SHM data are collected. By PF, the analytical joint distribution of states and parameters obtained by Bayes' Theorem is approximated through a discrete set of weighted particles, that represent random sample realizations in the joint states-parameters space [24]. Every time new data become available, the particles (states and parameters) are updated and further propagated forward in time by simulating the damage evolution model. Examples of PF-based approaches in the context of damage prognostics are found in $[15,25-29]$.

In addition, a general methodology for time-dependent reliability calculation is proposed based on filtered information about the future states of the system. This methodology is particularly useful for damage prognostics in composites where several damage modes may coexist, since reliability encapsulates information about the overall system performance. An approach for predicting time-dependent reliability has been 
adopted by $[17,30]$ in a similar manner, however in the present paper it is accomplished by PF focusing on damage in composites. In particular, it is shown that the time-dependent reliability calculation at a given time can be estimated as the sum of the normalized weights of the predicted particles that lie within a predefined useful domain, which is defined as the subregion of the state-space where system performance is authorized. Finally, a method for directly obtaining the RUL as a probability from the time-dependent reliability is proposed, whose validity is demonstrated using the axioms of Probability Logic [31, 32]. See Figure A.2 for a scheme of the proposed prediction framework based on reliability.

As a case study, the proposed prognostics approach is demonstrated using SHM data for matrix-crack density and stiffness reduction from a tension-tension fatigue experiment in a cross-ply CFRP laminate. Damage data are taken from NASA Ames Prognostics Data Repository (Composites dataset) [33]. Results show the suitability and potential of the proposed approach in performing RUL prediction with adequate management of the associated uncertainty.

The remainder of the paper is organized as follows. Section 2 discusses the theory behind fatigue damage in composites and presents the proposed methodology for fatigue damage modeling. The sequential state estimation problem by means of PF is presented in Section 3. Section 4 formally defines the prognostics problem and describes the methodology proposed to obtain the RUL estimation from the time-dependent reliability. In Section 5, the proposed framework is applied to a set of fatigue damage data to serve as an example. Finally, concluding remarks are provided in Section 6.

\section{Fatigue damage modeling}

The progression of fatigue damage in composites involves a progressive or sudden change of the macroscale mechanical properties, such as stiffness or strength, as a consequence of different fracture modes that evolve at the micro-scale along the lifespan of the structure [6]. In this work, the longitudinal stiffness loss is chosen as the macro-scale damage variable, given that, in contrast to the strength variable, it can be measured through in-situ non-destructive methods, which is of key importance for the filtering-based reliability approach proposed here. At the micro-scale level, matrix micro-cracking [34] is selected as the dominant fracture mode for the early stage of damage accumulation.

To accurately represent the relation between the internal damage and its manifestation through macroscale properties, several families of damage mechanics models have been proposed in the literature [35]. These models are based on first principles of admissible ply stress fields in presence of damage, and can be roughly classified into 1) analytical models, 2) semi-analytical models and 3) computational models. The two last families have been shown to be promising, however they are computationally prohibitive in a filtering-based prognostics approach, where a large amount of model evaluations is required. Therefore, the focus here is on the set of analytical models, that depending on the level of assumptions adopted to model the stress 
field in presence of damage, they can be classified (from simpler to more complex) into shear-lag models [36, 37], variational models [38], and crack opening displacement (COD) based models [39, 40]. Among them, the shear-lag models have received the most attention in the literature and, as a consequence, a vast number of extensions and variations can be found [35]. Shear-lag models use one-dimensional approximation of the equilibrium stress field after cracking to derive expressions for stiffness properties of the cracked laminate. The main modeling assumption of shear-lag models is that, in the position of matrix cracks, axial load is transferred to uncracked plies by the axial shear stresses at the interfaces. These models are usually restricted to cross-ply laminates or $\left[\phi_{\frac{n_{\phi}}{2}} / 90_{n_{90}} / \phi_{\frac{n_{\phi}}{2}}\right]$ lay-ups, where $\phi \in\left[-90^{\circ}, 90^{\circ}\right]$ is the ply-angle of the outer sublaminates (see Figure A.1). For general laminates with arbitrary stacking sequence, COD-based models are best suited. These models are expected to better capture the various damage mechanisms since they involve more complex damage mechanics, but it might be at expense of more information extracted from the data [41]. Then, if such models are utilized for future prediction, as in prognostics, the results are expected significantly dependent on the available data. However, it should be noted that the methodology proposed in this paper is not restricted to the above models but applicable to any damage modeling option.

In this work, the classical shear-lag model [36, 42] is the method chosen to represent the relation between the micro-cracks density and the stiffness loss, as it provides reasonable accuracy results while it depends less on the data quality. Therefore, it is expected to be less sensitive to the noise on data, as has been shown to hold true for composites materials by a recent study [41].

\subsection{Stiffness reduction model}

Following the unifying formulation by [43] for shear-lag models, the effective longitudinal Young's modulus $E_{x}^{*}$ can be calculated as a function of the crack-spacing in the $90^{\circ}$ layers as:

$$
E_{x}^{*}=\frac{E_{x, 0}}{1+a \frac{1}{2 l} R(\bar{l})}
$$

where $E_{x, 0}$ is the initial longitudinal Young's modulus of the undamaged laminate and $\bar{l}=\frac{l}{t_{90}}$ is the half crack-spacing normalized by the $90^{\circ}$ sublaminate thickness. The normalized half-crack spacing $\bar{l}$ can be expressed as a function of $\rho$, the matrix crack density, as: $\bar{l}=\frac{1}{2 \rho t_{90}}$. The term $a$ in Equation 1 is a function of mechanical and geometrical properties of the laminate as defined in Appendix A. The function $R(\bar{l})$, known as the average stress perturbation function, is defined as:

$$
R(\bar{l})=\frac{2}{\xi} \tanh (\xi \bar{l})
$$

where $\xi$, the shear-lag parameter, can be obtained for the classical shear-lag model [36, 42] as follows:

$$
\xi=\sqrt{G_{23}\left(\frac{1}{E_{2}}+\frac{t_{90}}{t_{\phi} E_{x}^{(\phi)}}\right)}
$$

The superscript $(\phi)$ denotes: "property of the $\left[\phi_{\frac{n_{\phi}}{2}}\right]$-sublaminate" (see Figure A.1 for further details). 


\subsection{Damage propagation model}

Having identified the model to express the relationship between the effective Young's modulus and microcracks density, the next step is to address the time evolution of the micro-cracks density. To this end, the shear-lag model is used to obtain the energy released per unit crack area due to the formation of a new crack between two existing cracks, denoted here by $G$. This energy, known as energy release rate (ERR), can be calculated as [44]:

$$
G=\frac{\sigma_{x}^{2} h}{2 \rho t_{90}}\left(\frac{1}{E_{x}^{*}(2 \rho)}-\frac{1}{E_{x}^{*}(\rho)}\right)
$$

where $\sigma_{x}$ is the applied axial tension, and $h$ and $t_{90}$ are the laminate and $90^{\circ}$ sublaminate half-thickness, respectively. The term $E_{x}^{*}(\rho)$, as a function of $\rho$, is the effective laminate Young's modulus due to the current damage state which can be calculated using Equation 1. The energy released calculated by Equation 4 can be further introduced into the modified Paris' Law [45] to obtain the evolution of matrix-cracks density as a function of fatigue cycle $n$, as shown below:

$$
\frac{d \rho}{d n}=A(\Delta G)^{\alpha}
$$

where $A$ and $\alpha$ are fitting parameters, and $\Delta G$ is the increment in ERR for a specific stress amplitude, i.e., $\Delta G=G\left(\sigma_{x, \max }\right)-G\left(\sigma_{x, \min }\right)$. Since the term $\Delta G$ involves the expression for the micro-damage mechanics model $E_{x}^{*}(\rho)$, a closed-form solution for Equation (5) is hard to obtain. To overcome this drawback, the resulting differential equation can be solved by approximating the derivative using unit-time finite differences, assuming that damage evolves cycle-to-cycle as:

$$
\rho_{n}=\rho_{n-1}+A\left(\Delta G\left(\rho_{n-1}\right)\right)^{\alpha}
$$

where $\rho_{n}$ is the matrix-cracks density at fatigue cycle $n \in \mathbb{N}$.

\section{Bayesian state estimation}

Having defined the model for damage propagation forward in time, the next step is to develop a method for sequential damage state estimation as new SHM data are collected. This is accomplished by Bayesian state estimation [46], that recursively uses Bayes' Theorem to incorporate the information from current SHM measurements along with the output of the damage propagation model, while accounting for the underlying uncertainties (e.g., modeling errors and measurement noise). To this end, a probabilistic description of the deterministic models described in Section 2 is required, which is developed in the next section. The Bayesian state estimation methodology is presented next. The proposed procedure will make extensive use of the concept of damage state, as a damage event predicted at a certain time or fatigue cycle, as explained below. 


\subsection{Stochastic system modeling}

Let us consider a generic damage progression model defined in state-space form using the following discrete state transition equation:

$$
\mathbf{x}_{n}=\mathbf{g}\left(\mathbf{x}_{n-1}, \mathbf{u}_{n}, \boldsymbol{\theta}\right)+\mathbf{v}_{n}
$$

where $\mathbf{g}\left(\mathbf{x}_{n-1}, \mathbf{u}_{n}, \boldsymbol{\theta}\right): \mathbb{R}^{n_{x}} \times \mathbb{R}^{n_{u}} \times \mathbb{R}^{n_{\theta}} \rightarrow \mathbb{R}^{n_{x}}$ is a possibly nonlinear function of the latent damage state $\mathbf{x}_{n} \in \mathbb{R}^{n_{x}}$ that may depend on a set of $n_{\theta}$ (uncertain) model parameters $\boldsymbol{\theta} \in \boldsymbol{\Theta} \subset \mathbb{R}^{n_{\theta}}$ along with a set of input parameters to the system $\mathbf{u}_{n} \in \mathbb{R}^{n_{u}}$. Here $\mathbf{v}_{n} \in \mathbb{R}^{n_{x}}$ is a model-error term that represents the difference between the actual system response $\mathbf{x}_{n}$ and the model output $\mathbf{g}$. This model error is assumed to be modeled as a zero-mean Gaussian distribution, which is supported by the Principle of Maximum Information Entropy (PMIE) [31, 47]. It follows that the entire state transition equation is also modeled as a Gaussian distribution, as:

$$
p\left(\mathbf{x}_{n} \mid \mathbf{x}_{n-1}, \mathbf{u}_{n}, \boldsymbol{\theta}\right)=\left((2 \pi)^{n_{x}}\left|\boldsymbol{\Sigma}_{\mathbf{v}_{n}}\right|\right)^{-\frac{1}{2}} \exp \left(-\frac{1}{2}\left(\mathbf{x}_{n}-\overline{\mathbf{x}}_{n}\right)^{T} \boldsymbol{\Sigma}_{\mathbf{v}_{n}}^{-1}\left(\mathbf{x}_{n}-\overline{\mathbf{x}}_{n}\right)\right)
$$

where $\overline{\mathbf{x}}_{n} \triangleq \mathbf{g}\left(\mathbf{x}_{n-1}, \mathbf{u}_{n}, \boldsymbol{\theta}\right)$, and $\boldsymbol{\Sigma}_{\mathbf{v}_{n}} \in \mathbb{R}^{n_{x} \times n_{x}}$ is the covariance matrix of the model error $\mathbf{v}_{n}$. Observe that the state transition equation, as defined in Equation 8, satisfies the Markov property [48], i.e., the modeled process is conditionally dependent on the past sequence only through the last state. Thus the proposed state transition equation describes a Markovian process of order one.

As discussed in Section 2, the progression of damage is studied in this paper by focusing on the matrixcracks density $\rho_{n}$, and the normalized effective stiffness $D_{n}=\frac{E_{x}^{*}}{E_{x, 0}}$, so that the following joint state transition equation of two components $\mathbf{g}=\left(g_{1}, g_{2}\right) \in \mathbb{R}^{2}$ is defined, as follows:

$$
\begin{gathered}
x_{1, n}=\rho_{n}=\underbrace{g_{1}\left(\rho_{n-1}, \mathbf{u}_{n}, \boldsymbol{\theta}\right)}_{\text {Eq. } 6}+v_{1, n} \\
x_{2, n}=D_{n}=\underbrace{g_{2}\left(\rho_{n}, \mathbf{u}_{n}, \boldsymbol{\theta}\right)}_{\text {Eq. } 1}+v_{2, n}
\end{gathered}
$$

where $\mathbf{x}_{n}=\left(x_{1, n}, x_{2, n}\right) \in \mathbb{R}^{2}$ is the system response at time $n \in \mathbb{N}$. Subscripts 1 and 2 correspond to the damage subsystems, namely, matrix-crack density and normalized effective stiffness, respectively. The vector $\mathbf{v}_{n}=\left(v_{1, n}, v_{2, n}\right) \in \mathbb{R}^{2}$ corresponds to the model error of the overall system. A key concept here is the consideration of model errors $v_{1, n}$ and $v_{2, n}$ as stochastically independent, even though the models corresponding to the damage subsystems, $g_{1}$ and $g_{2}$, are mathematically related, as shown in Section 2. It follows that the covariance operator $\boldsymbol{\Sigma}_{\mathbf{v}_{n}}$ is a diagonal matrix, i.e. $\boldsymbol{\Sigma}_{\mathbf{v}_{n}}=\operatorname{diag}\left(\sigma_{v_{1, n}}^{2}, \sigma_{v_{2, n}}^{2}\right)$, where $\sigma_{v_{1, n}}$ and $\sigma_{v_{2, n}}$ are the corresponding standard deviations of model errors $v_{1, n}$ and $v_{2, n}$, respectively. Therefore, the state transition equation of the overall system, as defined in Equation 8, can be readily expressed as a product of univariate 
Gaussians, as ${ }^{1}$ :

$$
p\left(\mathbf{x}_{n} \mid \mathbf{x}_{n-1}, \boldsymbol{\theta}\right)=p\left(D_{n} \mid \rho_{n}, \boldsymbol{\theta}\right) p\left(\rho_{n} \mid \rho_{n-1}, \boldsymbol{\theta}\right)
$$

where

$$
\begin{gathered}
p\left(\rho_{n} \mid \rho_{n-1}, \boldsymbol{\theta}\right)=\frac{1}{\sqrt{2 \pi} \sigma_{v_{1, n}}} \exp \left(-\frac{\left(\rho_{n}-g_{1}\left(\rho_{n-1}, \boldsymbol{\theta}\right)\right)^{2}}{2 \sigma_{v_{1, n}}^{2}}\right) \\
p\left(D_{n} \mid \rho_{n}, \boldsymbol{\theta}\right)=\frac{1}{\sqrt{2 \pi} \sigma_{v_{2, n}}} \exp \left(-\frac{\left(D_{n}-g_{2}\left(\rho_{n}, \boldsymbol{\theta}\right)\right)^{2}}{2 \sigma_{v_{2, n}}^{2}}\right)
\end{gathered}
$$

Let us now suppose that the system response can be measured during operation and that, at a certain fatigue cycle $n$, the measured system response can be expressed as a function of the latent state $\mathbf{x}_{n}$, as follows:

$$
\mathbf{y}_{n}=\mathbf{x}_{n}+\mathbf{w}_{n}
$$

where $\mathbf{y}_{n}=\left(y_{1, n}, y_{2, n}\right) \equiv\left(\hat{\rho}_{n}, \hat{D}_{n}\right) \in \mathbb{R}^{2}$ are the measurements of both, matrix-cracks density and normalized effective stiffness respectively, and $\mathbf{w}_{n}=\left(w_{1, n}, w_{2, n}\right) \in \mathbb{R}^{2}$ is the vector of measurement errors. As stated before, the PMIE is used to choose $\mathbf{w}_{n}$ as a zero mean Gaussian PDF with covariance matrix $\boldsymbol{\Sigma}_{\mathbf{w}_{n}}$. Thus, the measurement equation defined in Equation 12 can be expressed in probabilistic terms as

$$
p\left(\mathbf{y}_{n} \mid \mathbf{x}_{n}, \boldsymbol{\theta}\right)=\left((2 \pi)^{2}\left|\boldsymbol{\Sigma}_{\mathbf{w}_{n}}\right|\right)^{-\frac{1}{2}} \exp \left(-\frac{1}{2}\left(\mathbf{y}_{n}-\mathbf{x}_{n}\right)^{T} \boldsymbol{\Sigma}_{\mathbf{w}_{n}}^{-1}\left(\mathbf{y}_{n}-\mathbf{x}_{n}\right)\right)
$$

Since the measurements of each subsystem (micro-cracks and effective stiffness) are considered as stochastically independent, then $\boldsymbol{\Sigma}_{\mathbf{w}_{n}}=\operatorname{diag}\left(\sigma_{w_{1, n}}^{2}, \sigma_{w_{2, n}}^{2}\right)$, being $\sigma_{w_{1, n}}$ and $\sigma_{w_{2, n}}$ the standard deviation of the corresponding measurement errors $w_{1_{n}}$ and $w_{2_{n}}$, respectively. Thus, Equation 12 can be readily expressed as:

$$
p\left(\mathbf{y}_{n} \mid \mathbf{x}_{n}, \boldsymbol{\theta}\right)=p\left(\hat{\rho}_{n} \mid \rho_{n}\right) p\left(\hat{D}_{n} \mid D_{n}\right)
$$

where

$$
\begin{aligned}
p\left(\hat{\rho}_{n} \mid \rho_{n}\right) & =\frac{1}{\sqrt{2 \pi} \sigma_{w_{1, n}}} \exp \left(-\frac{\left(\hat{\rho}_{n}-\rho_{n}\right)^{2}}{2 \sigma_{w_{1, n}}^{2}}\right) \\
p\left(\hat{D}_{n} \mid D_{n}\right) & =\frac{1}{\sqrt{2 \pi} \sigma_{w_{2, n}}} \exp \left(-\frac{\left(\hat{D}_{n}-D_{n}\right)^{2}}{2 \sigma_{w_{2, n}}^{2}}\right)
\end{aligned}
$$

The PDFs for the state transition equation and the measurement equation defined in Equations 10 to 14 provide a complete statistical description of the system being modeled.

Finally, the set of uncertain model parameters $\boldsymbol{\theta}$ is selected among the complete set of mechanical and fitting parameters describing Equations 1 to 6 (see Table A.2) through a global sensitivity analysis [49]. The set of mechanical properties $\left\{E_{1}, E_{2}, t\right\}$ together with the Paris' Law's fitting parameter $\alpha$

\footnotetext{
${ }^{1}$ In what follows, the conditioning on the model input $\mathbf{u}_{n}$ is dropped for simpler notation.
} 
emerged as sensitive parameters to the model output uncertainty [41], so they are selected for sequential updating as shown below. Further, the standard deviations of the model errors $v_{1, n}$ and $v_{2, n}$ are added as candidates for updating since they are uncertain apriori, thereby resulting in the model parameter vector: $\boldsymbol{\theta}=\left(\alpha, E_{1}, E_{2}, t, \sigma_{v_{1, n}}, \sigma_{v_{2, n}}\right) \in \mathbb{R}^{6}$.

\subsection{Sequential state estimation}

The aim of sequential state estimation is to recursively estimate the posterior joint PDF of the latent system state $\mathbf{x}_{n}$ along with model parameters $\boldsymbol{\theta}$, at every time $n$ a new measurement is available. To this end, an augmented state $\mathbf{z}_{n}=\left\{\mathbf{x}_{n}, \boldsymbol{\theta}\right\}$ is defined in the joint state-parameter space $\mathcal{Z} \subset \mathbb{R}^{n_{x}} \times \mathbb{R}^{n_{\theta}}$, representing the overall system response including model parameters. Thus, given a sequence of measurements up to time $n, \mathbf{y}_{0: n} \triangleq\left\{\mathbf{y}_{0}, \mathbf{y}_{1}, \ldots, \mathbf{y}_{n-1}, \mathbf{y}_{n}\right\}$, the goal is to estimate the probability of the up-to-date sequence of latent states of the system $\mathbf{z}_{0: n} \triangleq\left\{\mathbf{z}_{0}, \mathbf{z}_{1}, \ldots, \mathbf{z}_{n-1}, \mathbf{z}_{n}\right\}$ through the conditional PDF $p\left(\mathbf{z}_{0: n} \mid \mathbf{y}_{0: n}\right)$. This is accomplished by Bayes' Theorem, as follows:

$$
\begin{aligned}
p\left(\mathbf{z}_{0: n} \mid \mathbf{y}_{0: n}\right) & =\frac{p\left(\mathbf{y}_{n} \mid \mathbf{z}_{n}\right) p\left(\mathbf{z}_{0: n} \mid \mathbf{y}_{0: n-1}\right)}{\int_{\mathcal{Z}} p\left(\mathbf{y}_{n} \mid \mathbf{z}_{n}\right) p\left(\mathbf{z}_{0: n} \mid \mathbf{y}_{0: n-1}\right) d \mathbf{z}_{0: n}} \\
& \propto p\left(\mathbf{y}_{n} \mid \mathbf{z}_{n}\right) p\left(\mathbf{z}_{n} \mid \mathbf{z}_{n-1}\right) \underbrace{p\left(\mathbf{z}_{0: n-1} \mid \mathbf{y}_{0: n-1}\right)}_{\text {last update }}
\end{aligned}
$$

where

$$
\begin{aligned}
p\left(\mathbf{z}_{n} \mid \mathbf{z}_{n-1}\right) & =\underbrace{p\left(\mathbf{x}_{n} \mid \mathbf{x}_{n-1}, \boldsymbol{\theta}_{n}\right)}_{\text {Eq. 10 }} p\left(\boldsymbol{\theta}_{n} \mid \boldsymbol{\theta}_{n-1}\right) \\
p\left(\mathbf{y}_{n} \mid \mathbf{z}_{n}\right) & =\underbrace{p\left(\mathbf{y}_{n} \mid \mathbf{x}_{n}, \boldsymbol{\theta}_{n}\right)}_{\text {Eq. 14 }}
\end{aligned}
$$

In Equation 16, it is assumed that $p\left(\mathbf{y}_{n} \mid \mathbf{z}_{0: n}, \mathbf{y}_{0: n-1}\right)=p\left(\mathbf{y}_{n} \mid \mathbf{z}_{n}\right)$ and that $p\left(\mathbf{z}_{n} \mid \mathbf{z}_{0: n-1}, \mathbf{y}_{0: n-1}\right)=p\left(\mathbf{z}_{n} \mid \mathbf{z}_{n-1}\right)$, based on the definition of the measurement equation (Equation 12) and the Markovian property of the state transition equation, respectively. It is also assumed that the initial state $\mathbf{z}_{0}$ is known in advance, hence $p\left(\mathbf{z}_{0} \mid \mathbf{y}_{0}\right) \equiv p\left(\mathbf{z}_{0}\right)$ (note that $\mathbf{y}_{0}$ is not a measurement), being $p\left(\mathbf{z}_{0}\right)=p\left(\mathbf{x}_{0} \mid \boldsymbol{\theta}\right) p(\boldsymbol{\theta})$ the prior PDF of the system state.

A key problem that typically arises when sequentially updating the state sequence $\mathbf{z}_{0: n}=\left\{\mathbf{x}_{0: n}, \boldsymbol{\theta}_{0: n}\right\}$ as an augmented state is the non-dynamics nature of $\boldsymbol{\theta}$, which makes it difficult to obtain the PDF $p\left(\boldsymbol{\theta}_{n} \mid \boldsymbol{\theta}_{n-1}\right)$ in Equation 16, and therefore, to explore the space of parameters $\boldsymbol{\Theta}$. A common solution is to add an independent random perturbation $\boldsymbol{\xi}_{n}$ to the set of updated parameters at time $n-1$ before evolving to the next predicted state at time $n$, i.e., $\boldsymbol{\theta}_{n}=\boldsymbol{\theta}_{n-1}+\boldsymbol{\xi}_{n}$. It induces a Markovian-type artificial dynamics [22, 50] to model parameters, whereby the PDF $p\left(\boldsymbol{\theta}_{n} \mid \boldsymbol{\theta}_{n-1}\right)$ is prescribed. For example, if $\boldsymbol{\xi}_{n}$ is assumed to be modeled as a zero-mean Gaussian, then the required PDF $p\left(\boldsymbol{\theta}_{n} \mid \boldsymbol{\theta}_{n-1}\right)$ is obtained as:

$$
p\left(\boldsymbol{\theta}_{n} \mid \boldsymbol{\theta}_{n-1}\right)=\mathcal{N}\left(\boldsymbol{\theta}_{n-1}, \Sigma_{\xi_{n}}\right)
$$


where $\Sigma_{\xi_{n}} \in \mathbb{R}^{n_{\theta} \times n_{\theta}}$ is the covariance matrix of the random walk which, in this work, is specified as $\Sigma_{\xi_{n}}=$ $\operatorname{diag}\left(\sigma_{\xi_{n, 1}}^{2}, \ldots, \sigma_{\xi_{n, j}}^{2}, \ldots, \sigma_{\xi_{n, n_{\theta}}^{2}}^{2}\right)$, i.e., each individual component of $\boldsymbol{\theta}$ performs an independent random walk, being $\sigma_{\xi_{n, j}}^{2}$ the variance of the random walk of $\theta_{n, j}$, the $j^{\text {th }}$ component the parameter vector $\boldsymbol{\theta}$. Observe that by this method, the model parameters are virtually time-evolving although they are essentially not dependent on time. In addition, note that such time-dependence imposes a loss of information in $\boldsymbol{\theta}$ over time (e.g., larger spread in $p\left(\boldsymbol{\theta}_{n} \mid \mathbf{y}_{0: n}\right)$ ) since additional uncertainties are artificially added to model parameters, which ultimately influences the precision of the state estimation. Several methods have been proposed in the literature to overcome this drawback, with the most popular being those that impose a shrinkage over $\Sigma_{\xi_{n}}$ as long as new data are collected [50]. An efficient method of this class has been recently proposed by Daigle and Goebel [51], which consists in modifying the variances $\sigma_{\xi_{n, j}}^{2}, j=1, \ldots, n_{\theta}$ by adding a negative scalar proportional to the relative distance between the actual and the target spread of the marginal posterior $p\left(\theta_{n, j} \mid \mathbf{y}_{0: n}\right)$, as follows:

$$
\sigma_{\xi_{n, j}}^{2}=\sigma_{\xi_{n-1, j}}^{2}\left(1-P_{j}^{*} \frac{\operatorname{RMAD}\left(\theta_{n, j}\right)-R M A D_{j}^{*}}{\operatorname{RMAD}\left(\theta_{n, j}\right)}\right)
$$

where $\operatorname{RMAD}\left(\theta_{n, j}\right)$ is the relative median absolute deviation of $p\left(\theta_{n, j} \mid \mathbf{y}_{0: n}\right), R M A D_{j}^{*}$ is the target $\operatorname{RMAD}$ for $p\left(\theta_{n, j} \mid \mathbf{y}_{0: n}\right)$, and $P_{j}^{*} \in[0,1]$ is a scaling constant that tunes the speed of convergence to $R M A D_{j}^{*}$. In [51], a comprehensive discussion about the optimal choice for $P_{j}^{*}$ and $R M A D_{j}^{*}$ is found. The term $\operatorname{RMAD}\left(\theta_{n, j}\right)$ can be readily calculated based on samples from the marginal posterior $p\left(\theta_{n, j} \mid \mathbf{y}_{0: n}\right)$, as follows:

$$
\operatorname{RMAD}\left(\theta_{n, j}\right)=\frac{\operatorname{median}\left(\left\{\left|\theta_{n, j}^{(k)}-\operatorname{median}\left(\tilde{\theta}_{n, j}\right)\right|\right\}_{k=1}^{K}\right)}{\operatorname{median}\left(\tilde{\theta}_{n, j}\right)}
$$

where $\tilde{\theta}_{n, j}=\left\{\theta_{n, j}^{(1)}, \ldots, \theta_{n, j}^{(k)}, \ldots, \theta_{n, j}^{(K)}\right\}$ is a set of $K$ samples from $p\left(\theta_{n, j} \mid \mathbf{y}_{0: n}\right)$. The adopted method for sequential parameter updating is summarized within Algorithm 1 below.

\subsection{Particle filters for joint state and parameter estimation}

The sequential state estimation methodology presented before requires the evaluation of multi-dimensional integrals of the type occurring in Equation 16, which are usually intractable except some especial cases using linear models and Gaussian uncertainties [23]. An alternative for the general case of both non-linear and non-Gaussian state-space models is by the use of particle methods [46], a set of sequential Monte Carlo methods which provide samples approximately distributed according to the posterior $\operatorname{PDF} p\left(\mathbf{z}_{0: n} \mid \mathbf{y}_{0: n}\right)$ with a feasible computational cost. Particle filters (PF) $[22,23]$ is one of the most common techniques among particle methods. With PF, a set of $N$ samples or particles with associated set of weights $\left\{\omega_{n}^{(i)}\right\}_{i=1}^{N}$, are used to obtain an approximation for the required posterior PDF as:

$$
p\left(\mathbf{z}_{0: n} \mid \mathbf{y}_{0: n}\right) \approx \sum_{i=1}^{N} \omega_{n}^{(i)} \delta\left(\mathbf{z}_{0: n}-\mathbf{z}_{0: n}^{(i)}\right)
$$


where $\delta$ is the Dirac delta. Given that the posterior density is seldom known exactly, it is not possible to obtain samples from it directly. For this reason, a sequential importance sampling (SIS) approach is adopted to straightforwardly generate samples from an importance density $q\left(\mathbf{z}_{0: n} \mid \mathbf{y}_{0: n}\right)$. Thus, to compensate for the difference between the importance density and the true posterior density, the unnormalized weights are computed as follows:

$$
\hat{\omega}_{n}^{(i)}=\frac{p\left(\mathbf{z}_{0: n}^{(i)} \mid \mathbf{y}_{0: n}\right)}{q\left(\mathbf{z}_{0: n}^{(i)} \mid \mathbf{y}_{0: n}\right)}
$$

where $\omega_{n}^{(i)}=\frac{\hat{\omega}_{n}^{(i)}}{\sum_{i=1}^{N} \hat{\omega}_{n}^{(i)}}, i=1, \ldots, N$. For practical reasons, the importance density is conveniently chosen as: $q\left(\mathbf{z}_{0: n} \mid \mathbf{y}_{0: n}\right)=q\left(\mathbf{z}_{0: n} \mid \mathbf{y}_{0: n-1}\right)$, therefore it can be factorized as $q\left(\mathbf{z}_{0: n} \mid \mathbf{y}_{0: n-1}\right)=q\left(\mathbf{z}_{n} \mid \mathbf{z}_{n-1}\right) q\left(\mathbf{z}_{0: n-1} \mid \mathbf{y}_{0: n-1}\right)$ [23]. Then, by substituting Equation 16 into Equation 22, the unnormalized importance weight for the $i^{\text {th }}$ particle at time $n$ can be rewritten as:

$$
\hat{\omega}_{n}^{(i)} \propto \underbrace{\frac{p\left(\mathbf{z}_{0: n-1}^{(i)} \mid \mathbf{y}_{0: n-1}\right)}{q\left(\mathbf{z}_{0: n-1}^{(i)} \mid \mathbf{y}_{0: n-1}\right)}}_{\omega_{n-1}^{(i)}} \frac{p\left(\mathbf{z}_{n}^{(i)} \mid \mathbf{z}_{n-1}^{(i)}\right) p\left(\mathbf{y}_{n} \mid \mathbf{z}_{n}^{(i)}\right)}{q\left(\mathbf{z}_{n}^{(i)} \mid \mathbf{z}_{n-1}^{(i)}\right)}
$$

Typically, the PDF $q\left(\mathbf{z}_{n} \mid \mathbf{z}_{n-1}\right)$ in Equation 23 is chosen to coincide to the state transition equation $p\left(\mathbf{z}_{n} \mid \mathbf{z}_{n-1}\right)$ since it is easy to evaluate $[22,52]$. By means of this, Equation 23 simplifies to:

$$
\hat{\omega}_{n}^{(i)} \propto \omega_{n-1}^{(i)} p\left(\mathbf{y}_{n} \mid \mathbf{z}_{n}^{(i)}\right)
$$

and the resulting algorithm is called bootstrap filter [22]. This is the algorithm adopted for the research in the present paper.

When particularizing to the fatigue problem investigated here, the data $\mathbf{y}_{0: n}$ are compounded by simultaneous measurements of both, micro-cracks density and normalized effective stiffness. Thus, by substituting the Equation 14 into 24, the formula for updating the particle weights leads to the next expression:

$$
\hat{\omega}_{n}^{(i)} \propto \omega_{n-1}^{(i)} p\left(\hat{\rho}_{n} \mid \rho_{n}^{(i)}\right) p\left(\hat{D}_{n} \mid D_{n}^{(i)}\right)
$$

A pseudocode implementation for the PF is provided as Algorithm 1. Observe that a systematic resampling step is implemented in Algorithm 1 to avoid the well-known drawback of weight degeneracy. During the resampling, particles are either dropped or reproduced that may result in a loss of diversity of the particles [23]. If necessary, a control step on this degeneracy by using the effective sample size (ESS) [53] may be incorporated before the resampling step.

It should be noted that when data are available over a set of non-regularly scheduled cycles $\{n, n+$ $k, \ldots, n+\ell\} \in \mathbb{N}$, with $\ell>k+1, k>1$, samples from the state transition equation $p\left(\mathbf{z}_{n+1} \mid \mathbf{z}_{n}\right)$ cannot be directly drawn. This is due to the one-step description of the matrix-cracks evolution model, as stated in Equation 9a. To overcome this drawback, which is usual in fatigue testing, the Total Probability Theorem can be applied to bridge the missing damage path growth between two non-subsequent measurements. 


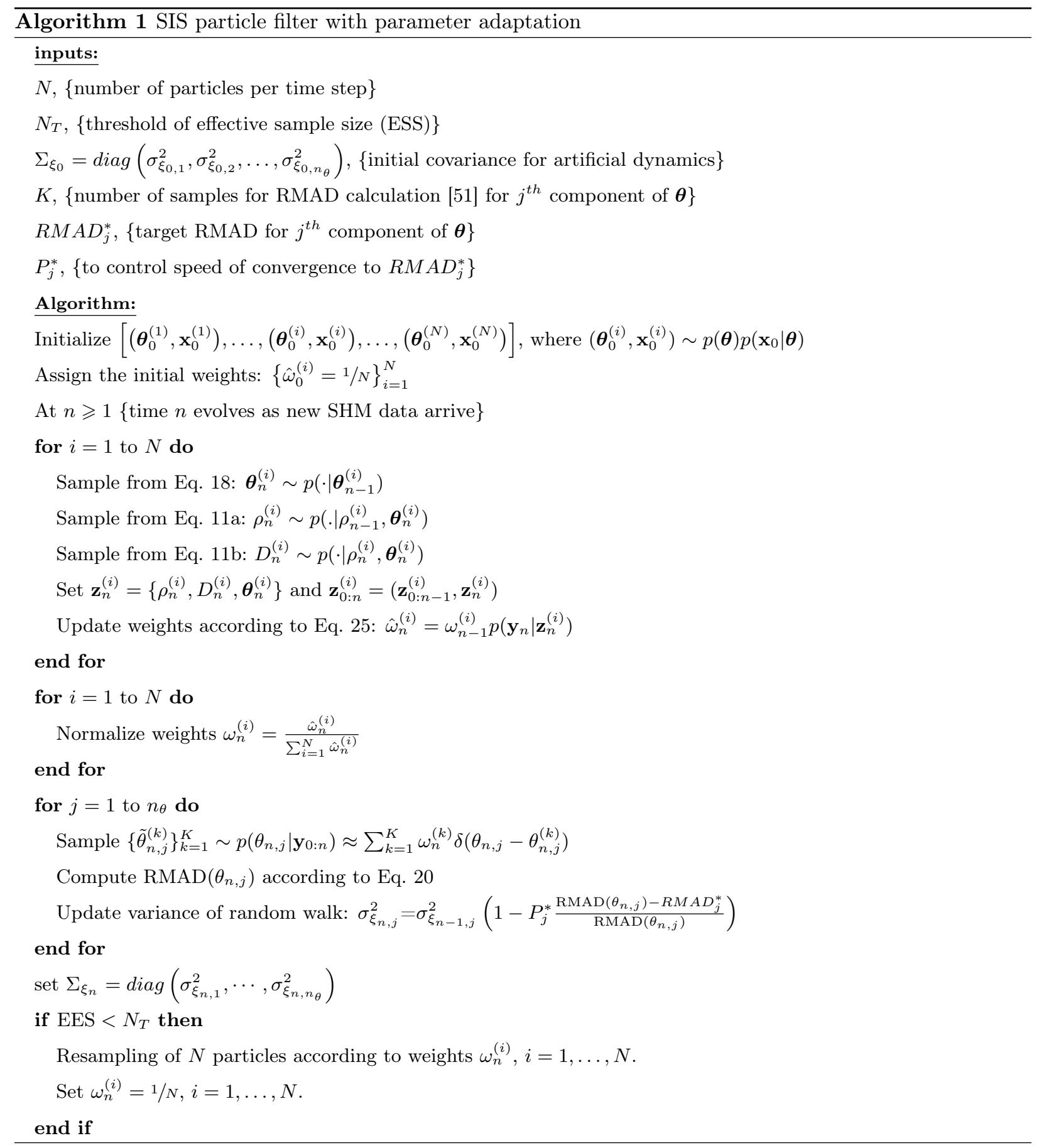


For example, for general cycles $n$ and $n+\ell$, with $\ell \in \mathbb{N} \geqslant 1$, the PDF $p\left(\mathbf{z}_{n+\ell} \mid \mathbf{z}_{n}\right)$ for the $\ell$-step-ahead states can be obtained as:

$$
p\left(\mathbf{z}_{n+\ell} \mid \mathbf{z}_{n}\right)=\int_{\mathcal{Z}} p\left(\mathbf{z}_{n+\ell} \mid \mathbf{z}_{n+1: n+\ell-1}, \mathbf{z}_{n}\right) p\left(\mathbf{z}_{n+1: n+\ell-1} \mid \mathbf{z}_{n}\right) d \mathbf{z}_{n+1: n+\ell-1}
$$

where $\mathbf{z}_{n+1: n+\ell-1}=\left\{\mathbf{z}_{n+1}, \mathbf{z}_{n+2}, \ldots, \mathbf{z}_{n+\ell-1}\right\} \in \mathcal{Z}$ is the sequence of missing states between the measuring times $n$ and $n+\ell$. Making use of the Markov property of state transition equation, Equation 27 can be rewritten as follows:

$$
p\left(\mathbf{z}_{n+\ell} \mid \mathbf{z}_{n}\right)=\int_{\mathcal{Z}} \prod_{t=n+1}^{n+\ell} p\left(\mathbf{z}_{t} \mid \mathbf{z}_{t-1}\right) d \mathbf{z}_{n+1: n+\ell-1}
$$

To numerically solve this multi-dimensional integral, an approximation can be readily obtained by conditional sampling, using recursively the one-step transition equation as defined in 10, i.e.: first sample $\mathbf{z}_{n+1}^{(i)}$ using the aforementioned one-step transition equation conditional on the initial state $\mathbf{z}_{n}$, i.e., $\mathbf{z}_{n+1}^{(i)} \sim p\left(\cdot \mid \mathbf{z}_{n}\right)$; then sample the succeeding state conditional on the previous sample, i.e., $\mathbf{z}_{n+2}^{(i)} \sim p\left(\cdot \mid \mathbf{z}_{n+1}^{(i)}\right)$; finally, repeat the same process until the target time $n+\ell$ is reached.

\subsection{Future state prediction}

Having estimated the probability distribution of the current damage state at time of prediction $n$, the next step for prognostics is to predict the distribution of future states of the system $\ell$-steps forward in time in absence of new observation, i.e., $p\left(\mathbf{z}_{n+\ell} \mid \mathbf{y}_{0: n}\right)$, with $\ell>1$. This distribution can be obtained by the Total Probability Theorem as:

$$
\begin{aligned}
p\left(\mathbf{z}_{n+\ell} \mid \mathbf{y}_{0: n}\right) & =\int_{\mathcal{Z}} p\left(\mathbf{z}_{n+\ell} \mid \mathbf{z}_{n: n+\ell-1}, \mathbf{y}_{0: n}\right) p\left(\mathbf{z}_{n: n+\ell-1} \mid \mathbf{y}_{0: n}\right) d \mathbf{z}_{n: n+\ell-1} \\
& =\int_{\mathcal{Z}}\left[\prod_{t=n+1}^{n+\ell} p\left(\mathbf{z}_{t} \mid \mathbf{z}_{t-1}\right)\right] p\left(\mathbf{z}_{n} \mid \mathbf{y}_{0: n}\right) d \mathbf{z}_{n: n+\ell-1}
\end{aligned}
$$

where $p\left(\mathbf{z}_{n} \mid \mathbf{y}_{0: n}\right)$ is the up-to-date information about the system at time $n$, and $p\left(\mathbf{z}_{t} \mid \mathbf{z}_{t-1}\right)$, with $t>n$, is the state transition equation which represents the future behavior of the system. It is important to remark here that, for simplicity but without loss of generality, input parameters $\mathbf{u}_{n}$ (e.g., loads, environmental conditions, etc.) are assumed to be known in advance and they are dropped from the formulation. Replacing $p\left(\mathbf{z}_{n} \mid \mathbf{y}_{0: n}\right)$ in Equation 28 by its particle-filter approximation (Equation 21), a particle estimation of the predictive $\operatorname{PDF} p\left(\mathbf{z}_{n+\ell} \mid \mathbf{y}_{0: n}\right)$ can be obtained as:

$$
\begin{aligned}
p\left(\mathbf{z}_{n+\ell} \mid \mathbf{y}_{0: n}\right) & \approx \int_{\mathcal{Z}}\left[\prod_{t=n+1}^{n+\ell} p\left(\mathbf{z}_{t} \mid \mathbf{z}_{t-1}\right)\right] \sum_{i=1}^{N} \omega_{n}^{(i)} \delta\left(\mathbf{z}_{0: n}-\mathbf{z}_{0: n}^{(i)}\right) d \mathbf{z}_{n: n+l-1} \\
& =\sum_{i=1}^{N} \omega_{n}^{(i)} \int_{\mathcal{Z}} p\left(\mathbf{z}_{n+1} \mid \mathbf{z}_{n}^{(i)}\right) \prod_{t=n+2}^{n+\ell} p\left(\mathbf{z}_{t} \mid \mathbf{z}_{t-1}\right) d \mathbf{z}_{n+1: n+\ell-1}
\end{aligned}
$$


Note that last equation cannot be solved analytically, however it can be sampled by drawing one conditional sample sequence $\mathbf{z}_{n+1: n+\ell}^{(i)}=\left\{\mathbf{z}_{n+1}^{(i)}, \mathbf{z}_{n+2}^{(i)}, \ldots, \mathbf{z}_{n+\ell}^{(i)}\right\}$ from each of the $N$ multidimensional integrals in Equation 29, using the conditional sampling methodology explained in last section. Each of the simulated sequences $\mathbf{z}_{n+1: n+\ell}^{(i)}, i=1, \cdots, N$ adopts the weight $\omega_{n}^{(i)}$ of the corresponding "stem" sample $\mathbf{z}_{n}^{(i)}$; therefore an approximation of the $\ell$-step predictive ahead PDF can be obtained as:

$$
p\left(\mathbf{z}_{n+\ell} \mid \mathbf{y}_{0: n}\right) \approx \sum_{i=1}^{N} \omega_{n}^{(i)} \delta\left(\mathbf{z}_{n+\ell}-\mathbf{z}_{n+\ell}^{(i)}\right)
$$

where $\mathbf{z}_{n+\ell}^{(i)} \in \mathbf{z}_{n+1: n+\ell}^{(i)}, \quad i=1 \ldots N$.

\section{Reliability based prognostics}

Reliability is a probabilistic metric that provides information about the system performance in relation to a predefined limit state or threshold function. When the states of the system under study are time-dependent, as in the case of fatigue damage accumulation in composite materials, then the reliability calculation may depend upon the health state of the system at a generic time instant, leading to the concept of time-dependent reliability $[30,54,55]$. In this context, it is possible to formulate the problem of $\ell$-step-ahead prediction of reliability, denoted here as $R_{n+\ell \mid n}$, using the most up-to-date information about the system at time $n$. It is further shown in this section that the predicted time-dependent reliability also serves to derive a cumulative distribution function (CDF) of the RUL in a straightforward manner.

\subsection{Time-dependent reliability}

Let us define the useful domain $\mathcal{U} \subset \mathcal{Z}$ as the non-empty subset of authorized states of the system, and the complementary subset $\overline{\mathcal{U}}=\mathcal{Z} \backslash \mathcal{U}$, as the subset of states where the system behavior becomes unacceptable, or simply, where system failure occurs. Then, the time-dependent reliability can be defined as the probability ${ }^{2}$ of the system to belong to the useful domain $\mathcal{U}$ at general time $n+\ell$, based on updated information about the system states at cycle $n$. In mathematical terms:

$$
R_{n+\ell \mid n}=P\left(\mathbf{z}_{n+\ell} \in \mathcal{U} \mid \mathbf{y}_{0: n}\right)=\int_{\mathcal{U}} p\left(\mathbf{z}_{n+\ell} \mid \mathbf{y}_{0: n}\right) d \mathbf{z}_{n+\ell}
$$

where $p\left(\mathbf{z}_{n+\ell} \mid \mathbf{y}_{0: n}\right)$ is the $\ell$-step ahead predictive PDF of the system, as defined in Equation 28. The last probability integral, defined over the useful domain $\mathcal{U}$, can be redefined over the complete $\mathbf{z}$-space by introducing an indicator function $\mathbb{I}_{(\mathcal{U})}(\mathbf{z})$, as follows:

$$
R_{n+\ell \mid n}=\int_{\mathcal{Z}} \mathbb{I}_{(\mathcal{U})}\left(\mathbf{z}_{n+\ell}\right) p\left(\mathbf{z}_{n+\ell} \mid \mathbf{y}_{0: n}\right) d \mathbf{z}_{n+\ell}
$$

\footnotetext{
${ }^{2}$ In what follows, $P(\cdot)$ is used to denote probability, whereas a PDF is expressed as $p(\cdot)$.
} 
where $\mathbb{I}_{(\mathcal{U})}(\mathbf{z}): \mathcal{Z} \rightarrow\{0,1\}$ maps a given point in the joint state-parameter space $\mathcal{Z}$ to the Boolean domain $\{0,1\}$, such that:

$$
\mathbb{I}_{(\mathcal{U})}(\mathbf{z})= \begin{cases}1, & \text { if } \mathbf{z} \in \mathcal{U} \\ 0, & \text { if } \mathbf{z} \in \overline{\mathcal{U}}\end{cases}
$$

By replacing $p\left(\mathbf{z}_{n+\ell} \mid \mathbf{y}_{0: n}\right)$ by its future estimate given by Equation 30, an estimate of the integral in Equation 32 can be obtained as:

$$
\begin{aligned}
R_{n+\ell \mid n} & \approx \int_{\mathcal{Z}} \mathbb{I}_{(\mathcal{U})}\left(\mathbf{z}_{n+\ell}\right)\left[\sum_{i=1}^{N} \omega_{n}^{(i)} \delta\left(\mathbf{z}_{n+\ell}-\mathbf{z}_{n+\ell}^{(i)}\right)\right] d \mathbf{z}_{n+\ell} \\
& =\sum_{i=1}^{N} \omega_{n}^{(i)} \mathbb{I}_{(\mathcal{U})}\left(\mathbf{z}_{n+\ell}^{(i)}\right)
\end{aligned}
$$

From the last equation, it is shown that the $\ell$-step ahead predicted reliability of the system can be readily approximated as the sum of the weights of the subset of particles that satisfy $\mathbb{I}_{(\mathcal{U})}\left(\mathbf{z}_{n+\ell}^{(i)}\right)=1$, i.e., those that lie within the useful domain at time $n+\ell$. By evaluating Equation 34 for different values $\ell \geqslant 1$, a time dependent reliability function is obtained. Note that, as a particular case of the time-dependent reliability, an estimation of the updated reliability $R_{n \mid n}$ can be obtained at time $n$ (when the last SHM measurement is available) as:

$$
R_{n \mid n}=\sum_{i=1}^{N} \omega_{n}^{(i)} \mathbb{I}_{(\mathcal{U})}\left(\mathbf{z}_{n}^{(i)}\right)
$$

where $\left\{\mathbf{z}_{n}^{(i)}, \omega_{n}^{(i)}\right\}_{i=1}^{N}$ is a set of $N$ discrete samples to approximate the PDF $p\left(\mathbf{z}_{n} \mid \mathbf{y}_{0: n}\right)$.

\subsection{Calculation of RUL based on time-dependent reliability}

Once the problem of future state prediction has been assessed and the time-dependent reliability of the system has been derived, the next natural step for prognostics is to estimate the remaining useful life of the engineering component/system; i.e., to estimate the minimum time $\ell$ when the predicted state is expected to lie within the failure domain $\overline{\mathcal{U}}$. In mathematical terms:

$$
R U L_{n}=\inf \left\{\ell \in \mathbb{N}: \mathbf{z}_{n+\ell} \in \overline{\mathcal{U}}\right\}
$$

In the context of the fatigue problem investigated in this paper, the $\mathrm{RUL}_{n}$ corresponds to the minimum amount of prospective fatigue cycles starting from $n$, such that damage (matrix-cracks or stiffness loss) goes beyond a predefined damage threshold, that is defined as the boundary of the useful domain $\mathcal{U}$. Observe that, based on the definition in Equation 36, it is clear that the proposition $\left[R U L_{n} \leqslant \ell\right]$ implies the proposition $\left[\mathbf{z}_{n+\ell} \in \overline{\mathcal{U}}\right]$ and vice versa, i.e., $\left[R U L_{n} \leqslant \ell\right] \Longleftrightarrow\left[\mathbf{z}_{n+\ell} \in \overline{\mathcal{U}}\right]$. However, it is necessary to further explore the correspondence between both propositions in terms of probability, which would allow us a direct connection between the RUL and time dependent reliability. In the next subsection, the equivalence between $P\left(R U L_{n} \leqslant\right.$ $\left.\ell \mid \mathbf{y}_{0: n}\right)$ and $P\left(\mathbf{z}_{n+\ell} \in \overline{\mathcal{U}} \mid \mathbf{y}_{0: n}\right)$ is derived and examined under the axioms of Probability Logic [31, 32]. 


\subsubsection{Derivation of probability of RUL from Probability Logic}

In Probability Logic, $P(b \mid a)$ is interpreted as the degree of plausibility of proposition $b$ based on the information given by proposition $a$ [32]. In other words, given the proposition $a$, then proposition $b$ holds with probability $P(b \mid a)$. In the specific situation when then proposition $b$ gives complete information about $a$, i.e. $b \Longrightarrow a$, then $P(a \mid b)=1$. By the contrary, when $b$ implies not $a$, then $P(a \mid b)=0$. Four axioms are defined in Probability Logic:

$$
\begin{aligned}
P(b \mid a) & \geqslant 0 \\
P(b \mid a)+P(\sim b \mid a) & =1 \\
P(b \mid b \& a) & =1 \\
P(c \& b \mid a) & =P(c \mid b \& a) P(b \mid a)
\end{aligned}
$$

where $\sim b$ reads "not $b$ " and $a \& b$ reads " $a$ and $b$ ". From these axioms, the property $P(b \mid a) \leqslant 1$ is obtained, which can be readily derived from axioms (37a) and (37b).

Let us now suppose that proposition $a$ represents the data $\mathbf{y}_{0: n}, b$ represents $\left[R U L_{n} \leqslant \ell\right]$, and $c$ represents $\left[\mathbf{z}_{n+\ell} \in \overline{\mathcal{U}}\right]$. As evident from the definition in Equation 36, the proposition $\left[R U L_{n} \leqslant \ell\right]$ implies the proposition $\left[\mathbf{z}_{n+\ell} \in \overline{\mathcal{U}}\right]$ and vice versa, i.e., $b \Longleftrightarrow c$. Next, from axiom $37 \mathrm{~d}$ :

$$
\begin{aligned}
P(b \& c \mid a) & =P(b \mid c \& a) P(c \mid a) \\
& =\underbrace{P(b \mid(b \Longleftrightarrow c) \& a)}_{=1, \text { by axiom }(37 c)} P(c \mid a)
\end{aligned}
$$

where the equivalence $c \& a \equiv(b \Longleftrightarrow c) \& a$ is used in Equation 38b. Thus $P(b \& c \mid a)=P(c \mid a)$ and also $P(b \& c \mid a)=P(b \mid a)$, based on the correspondence $b \Longleftrightarrow c$. The latter formally proves the following equality in terms of probabilities: $P\left(R U L_{n} \leqslant \ell \mid \mathbf{y}_{0: n}\right)=P\left(\mathbf{z}_{n+\ell} \in \overline{\mathcal{U}} \mid \mathbf{y}_{0: n}\right)$.

\subsection{Prognostics based on time-dependent reliability}

The reasoning given above allows us to establish a rational connection between the RUL as a probability, and the time-dependent reliability, provided that the events $\left[\mathbf{z}_{n+\ell} \in \overline{\mathcal{U}}\right]$ and $\left[R U L_{n} \leqslant \ell\right]$ occur with the same probability; hence the following identities hold:

$$
\begin{aligned}
F_{R U L_{n}}(\ell-n) & \triangleq P\left(R U L_{n} \leqslant \ell \mid \mathbf{y}_{0: n}\right)=P\left(\mathbf{z}_{n+\ell} \in \overline{\mathcal{U}} \mid \mathbf{y}_{0: n}\right) \\
& =1-P\left(\mathbf{z}_{n+\ell} \in \mathcal{U} \mid \mathbf{y}_{0: n}\right) \\
& =1-R_{n+\ell \mid n}
\end{aligned}
$$

which makes use of the definition of time-dependent reliability given in Equation 31. In the last equation, $F_{R U L_{n}}(\ell-n)$ denotes the CDF of the random variable $\ell-n \in \mathbb{N}$. Thus, the probability $P\left(R U L_{n} \leqslant \ell \mid \mathbf{y}_{0: n}\right)$ 
can be approximated using Equation 34 as:

$$
P\left(R U L_{n} \leqslant \ell \mid \mathbf{y}_{0: n}\right) \approx 1-\sum_{i=1}^{N} \omega_{n}^{(i)} \mathbb{I}_{(\mathcal{U})}\left(\mathbf{z}_{n+\ell}^{(i)}\right)
$$

Observe that it is possible to compute the entire CDF of $R U L_{n}$ by evaluating Equation 40 for different values of $\ell \geqslant 1$ until the value $R_{n+\ell \mid n}=0$ is reached, which coincides when $F_{R U L_{n}}(\ell-n)=1$. See Figure A.2 for a scheme of the proposed reliability-based prognostics framework. The calculation of the time-dependent reliability can be updated each time $n$ new data are collected. The outcomes of these steps are conceptually illustrated in Figure A.3.

\section{Case study}

The proposed framework is applied to fatigue cycling data obtained from a set of run-to-failure fatigue experiments in cross-ply graphite-epoxy laminates. Torayca T700G unidirectional pre-impregnated (commonly known as prepreg) material was used for $15.24[\mathrm{~cm}] \times 25.4[\mathrm{~cm}]$ coupons with dogbone geometry and $\left[0_{2} / 90_{4}\right]_{\mathrm{s}}$ stacking sequence. A notch $(5.1[\mathrm{~mm}] \times 19.3[\mathrm{~mm}])$ was created in these coupons to induce damage modes other than matrix-cracks, such as delamination, thereby introducing additional sources of uncertainty and then demonstrating the proposed framework under more realistic conditions. The main mechanical properties of such coupons are listed in Table A.2.

The tests were conducted under load-controlled tension-tension cyclic loading with a maximum applied load of 31.13 [KN], a frequency $f=5[\mathrm{~Hz}]$, and a stress ratio $R=0.14$ (relation between the minimum and maximum stress for each cycle). Monitoring data were collected from a network of 12 piezoelectric (PZT) sensors using Lamb wave signals and three triaxial strain-gages. Both micro-crack density and stiffness reduction data were considered as NDE measurements during the fatigue test. The mapping between PZT raw data and micro-cracks density was done following the methodology proposed in [56]. More details about these tests are reported in [33, 57]. Damage data used in this example correspond to laminate L1S19 in [33] (see a summary in Table A.1). Figure A.4 provides a schematic view of the experimental set-up.

Results for sequential state estimation along with multi-step ahead prediction for both micro-cracks density and normalized effective stiffness are presented for three different time instants (cycles) in Figure A.5. Note that at the beginning of each plot (left side before the multi-step ahead prediction) the collected data up to cycle $n, n=\{1,4,8\} \times 10^{4}$ are represented along with the sequence of filtered states, which are estimated using Algorithm 1 with $N=5000$ particles. For this case study, the systematic importance resampling (SIR) variant of the SIS algorithm is adopted, whereby the resampling step is run every time new data are collected. Damage states are initialized at $\mathbf{x}_{0}=\left(\rho_{0}, D_{0}\right)$, being $\rho_{0}=0.1[\mathrm{cracks} / \mathrm{mm}]$ and $D_{0}=1$ (dimensionless). The standard deviation of the measurement error parameters are set to $\sigma_{w_{1, n}}=$ $0.05[$ cracks $/ m m]$ and $\sigma_{w_{2, n}}=0.01$, taking them as known. The scaling variables $R M A D_{j}^{*}$ and $P_{j}^{*}$ are fixed 
to $0.3 \cdot R M A D_{0, j}$ and 0.001 respectively, where $R M A D_{0, j}$ is calculated according to Equation 20 using samples from the marginal prior $\operatorname{PDF} p\left(\theta_{j}\right), j=1, \ldots, 6$. The chosen prior PDFs for model parameters $\boldsymbol{\theta}=\left\{\theta_{1}, \theta_{2}, \ldots \theta_{6}\right\}$ are specified in Table A.2. The diagonal elements $\sigma_{\xi_{0, j}}$ of the covariance matrix $\Sigma_{\xi_{0}}$ (recall Equation 18) are appropriately selected through initial test runs and set to $0.5 \%$ of the $5^{\text {th }}-95^{\text {th }}$ inter percentile range of the marginal priors $p\left(\theta_{j}\right), j=1, \ldots, 6$. To reveal the uncertainty reduction in model parameters $\boldsymbol{\theta}$, the posterior mean of the $j^{\text {th }}$ component is plotted against cycles in Figure A.6 for $j=$ $1, \ldots, 6$, as well as their $25 \%-75 \%, 5 \%-95 \%$ probability bands.

Moreover, time-dependent reliability estimations are obtained using the methodology described in Section 4.1. The results are shown in Figure A.7 for selected cycles $n=\{0.1,1,3,5,7,9\} \times 10^{4}$. For this example, the useful domain is defined by the subset $\mathcal{U}=\{(\rho, D) \in[0,0.418] \times[1,0.88]\} \subset \mathbb{R}^{2}$, where $\rho$ is expressed in $[$ cracks $/ \mathrm{mm}]$ and $D$ is dimensionless. By comparing between consecutive plots in Figure A.7, one can observe that the reliability prediction gradually tends to converge as more SHM data become available.

The RUL calculated from the predicted reliability is shown in Figure A.8, where two cones of accuracy at $10 \%$ and $20 \%$ of the true RUL (denoted as RUL*) are drawn to help evaluating the prediction accuracy and precision [58]. Observe that the RUL prediction is appreciably inaccurate for the initial stages of the fatigue process, which suggests that a number of cycles are required for SHM data to train model parameters. From this period, not only the prediction precision clearly improves with time (values closer to RUL* line), but also the prediction spread gradually tends to diminish. Observe also that from cycle $n=5 \cdot 10^{4}$, the estimated mean values for the RUL (labelled by the circles in Figure A.8) get higher values with respect to the RUL* line, progressively leaving the accuracy area. However, the median RUL estimates (labelled by the squares) remain within the accuracy region. An explanation for this observation is provided in view of the asymptotic behavior of the damage process for both, micro-cracks density and normalized stiffness decrease, as shown in Figure A.5. Note that from cycle $n=5 \cdot 10^{4}$, the model produces a large amount of predicted samples that already lie within the failure domain $\overline{\mathcal{U}}$ at time of prediction $n$. This leads to an increasing density of predicted $\mathrm{RUL}_{n}$ concentrated at cycle $n$, in addition to a distributional tail of $\mathrm{RUL}_{n}$ corresponding to those particles that have not reached the failure region at cycle $n$. These particles hit the failure threshold at cycles much higher than $n$, as a consequence of the asymptotic damage progression. Thus, the predicted mean values of RUL show a positive shift with respect to the RUL* values, whereas the median RUL estimates remain closer to RUL*. The last observation is clearly manifested at $n=9 \cdot 10^{4}$, which corresponds to the cycle when the measured damage reaches the failure region. Observe in Figure A.8 that the estimation of $\mathrm{RUL}_{n}$ at $n=9 \cdot 10^{4}$ displays an upper distributional tail, that makes sense with the updated reliability value $R_{n \mid n}=0.42$ at that cycle, meaning that there is up-to a $42 \%$ of remaining reliability for cycles $n \geqslant 9 \cdot 10^{4}$. 


\section{Concluding remarks}

A prediction methodology based on reliability was proposed to obtain the remaining useful life of composites under fatigue conditions. The remaining useful life was derived from a propagation of the time-dependent reliability. Physics-based models were considered to predict the future evolution of damage, due to the benefits for predicting reliability and RUL. Two damage variables, micro-cracks density and stiffness loss, were simultaneously considered to represent the health state of the laminate. The validity of this framework was demonstrated on SHM data collected from a tension-tension fatigue experiment using a CFRP cross-ply laminate. Reliability, as defined in this work, emerged as a suitable unified system-health indicator for prognostics as it encapsulates information about the system health state while it allows predicting the RUL of the system. More research effort is needed to develop more efficient prognostics algorithms to improve the accuracy at the final stage of the process, where damage typically reaches an asymptotic behavior, and to extend this prognostics framework from coupon level to component or subsystem levels.

\section{Acknowledgment}

The two first authors would like to thank the Ministry of Education of Spain for the FPU grants AP20094641, AP2009-2390 and the Prognostics Center of Excellence at NASA Ames Research Center, which kindly hosted them during the course of this work. Authors would also like to thank the Structures and Composites lab at Stanford University for experimental data and NASA ARMD/AvSafe project SSAT, which provided partial support for this work.

\section{Appendix A. Nomenclature and basic relations}

For ply and laminate properties, the nomenclature exposed in Table A.3 is adopted in this work. Notice that the subscripts $\{1,2,3\}$ refer to ply properties defined in local axes while the subscripts $\{x, y, z\}$ refer to sublaminate or laminate properties defined in global axes, that corresponds to the laminate coordinate system (see Figure A.1b). The first local direction "1" coincides with fibers direction at a given ply or lamina (on-axis direction), while directions "2-3" are the in-plane and out-of-plane transverse directions. For global axes, "x" refers to the fatigue loading direction, while "y-z" refers to the in-plane and out-of-plane transverse directions, respectively. In addition, the superscript $(\phi)$ denotes: "property of the $\left[\phi_{\frac{n_{\phi}}{2}}\right]$-sublaminate".

The function $a$ in Equation 1 is defined as a function of the laminate and ply properties as follows:

$$
a=\frac{E_{2} t_{90}}{E_{1} t_{\phi}}\left(1-\nu_{x y}^{(\phi)} \frac{\frac{\nu_{x y}^{(\phi)} t_{90}}{E_{y}^{(\phi)}}+\frac{\nu_{12} t_{\phi}}{E_{2}}}{\frac{t_{90}}{E_{y}^{(\phi)}}+\frac{t_{\phi}}{E_{1}}}\right) \frac{1-\nu_{12} \nu_{x y}^{(\phi)}}{1-\nu_{12}^{2} \frac{E_{2}}{E_{1}}}
$$


where properties with the superscript $(\phi)$ are referred to the outer $\left[\phi_{\frac{n_{\phi}}{2}}\right]$-sublaminate. From the classical theory of laminates [59], these properties can be readily obtained as:

$$
\begin{aligned}
& \frac{1}{E_{x}^{(\phi)}}=\frac{m^{4}}{E_{1}}+\frac{n^{4}}{E_{2}}+\left(\frac{1}{G_{12}}-2 \frac{\nu_{12}}{E_{1}}\right) m^{2} n^{2} \\
& \frac{1}{E_{y}^{(\phi)}}=\frac{n^{4}}{E_{1}}+\frac{m^{4}}{E_{2}}+\left(\frac{1}{G_{12}}-2 \frac{\nu_{12}}{E_{1}}\right) m^{2} n^{2} \\
& \frac{\nu_{x y}^{(\phi)}}{E_{x}^{(\phi)}}=\frac{\nu_{12}}{E_{1}}-\left(\frac{1+2 \nu_{12}}{E_{1}}+\frac{1}{E_{2}}-\frac{1}{G_{12}}\right) m^{2} n^{2}
\end{aligned}
$$

where $m=\cos (\phi)$ and $n=\sin (\phi)$, and $\phi$ is the angle between the laminate $x$-axis and the fiber direction of $\left[\phi_{\frac{n_{\phi}}{2}}\right]$-sublaminate, as Figure A.1b illustrates. The rest of the parameters involved in Equations A.1 and A.2 are defined in Table A.3. For cross-ply laminates, as the laminate type considered in Section $5, \phi=0^{\circ}$, thus the laminate and sublaminate global axes $\{x, y, z\}$ coincide with ply local axes $\{1,2,3\}$. In this particular case, the following identities hold:

$$
E_{x}^{(0)}=E_{1} ; \quad E_{y}^{(0)}=E_{2} ; \quad \nu_{x y}^{(0)}=\nu_{12} ; \quad G_{x y}^{(0)}=G_{12} ; \quad G_{x z}^{(0)}=G_{12}
$$

The undamaged longitudinal Young's modulus of the overall laminate, $E_{x, 0}$, can be obtained as $E_{x, 0}=$ $\frac{1}{a_{11}^{*}}$, where $a_{11}^{*}$ is the $(1,1)^{t h}$ element of $\mathbf{a}^{*}$, the normalized compliance matrix of the laminate. The matrix $\mathbf{a}^{*}$ can be obtained as the inverse of the normalized laminate stiffness matrix $\mathbf{A}^{*}$, i.e., $\mathbf{a}^{*}=\left(\mathbf{A}^{*}\right)^{-1}$. For the laminate type considered in this work, the stiffness matrix $\mathbf{A}^{*}$ can be readily calculated using the rule of mixtures as $\mathbf{A}^{*}=\frac{t_{\phi}}{h} \overline{\mathbf{Q}}^{(\phi)}+\frac{t_{90}}{h} \overline{\mathbf{Q}}^{(90)}$, where $\overline{\mathbf{Q}}^{(\alpha)}, \alpha=\{\phi, 90\}$ is the corresponding stiffness matrix of the outer $\left[\phi_{\frac{n_{\phi}}{2}}\right]$-sublaminates and 90-sublaminate, respectively, defined as:

$$
\overline{\mathbf{Q}}^{(\alpha)}=\left[\begin{array}{lll}
\bar{Q}_{11} & \bar{Q}_{12} & \bar{Q}_{16} \\
\bar{Q}_{21} & \bar{Q}_{22} & \bar{Q}_{62} \\
\bar{Q}_{61} & \bar{Q}_{62} & \bar{Q}_{66}
\end{array}\right]
$$

The elements of this matrix can be obtained as a function of the corresponding sublaminate angle $\alpha$ as:

$$
\left[\begin{array}{l}
\bar{Q}_{11} \\
\bar{Q}_{22} \\
\bar{Q}_{12} \\
\bar{Q}_{66} \\
\bar{Q}_{16} \\
\bar{Q}_{26}
\end{array}\right]=\left[\begin{array}{rrrrr}
U_{1} & U_{2} & U_{3} & 0 & 0 \\
U_{1} & -U_{2} & U_{3} & 0 & 0 \\
U_{4} & 0 & -U_{3} & 0 & 0 \\
U_{5} & 0 & -U_{3} & 0 & 0 \\
0 & 0 & 0 & \frac{1}{2} U_{2} & U_{3} \\
0 & 0 & 0 & \frac{1}{2} U_{2} & -U_{3}
\end{array}\right] \cdot\left[\begin{array}{r}
1 \\
\cos (2 \alpha) \\
\cos (4 \alpha) \\
\sin (2 \alpha) \\
\sin (4 \alpha)
\end{array}\right]
$$

where $U_{i}, i=1, \ldots, 5$ are invariants of the ply, whose values are defined regardless of the ply orientation as 
a function of the components of the on-axis ply stiffness matrix, as follows:

$$
\begin{aligned}
U_{1} & =\frac{3}{8} Q_{11}+\frac{3}{8} Q_{22}+\frac{1}{4} Q_{12}+\frac{1}{2} Q_{66} \\
U_{2} & =\frac{1}{2} Q_{11}-\frac{1}{2} Q_{22} \\
U_{3} & =\frac{1}{8} Q_{11}+\frac{1}{8} Q_{22}-\frac{1}{4} Q_{12}-\frac{1}{2} Q_{66} \\
U_{4} & =\frac{1}{8} Q_{11}+\frac{1}{8} Q_{22}+\frac{3}{4} Q_{12}-\frac{1}{2} Q_{66} \\
U_{5} & =\frac{1}{8} Q_{11}+\frac{1}{8} Q_{22}-\frac{1}{4} Q_{12}+\frac{1}{2} Q_{66}
\end{aligned}
$$

where

$$
Q_{11}=\frac{E_{1}}{1-\nu_{12}^{2} \frac{E_{2}}{E_{1}}}, \quad Q_{22}=\frac{E_{2}}{1-\nu_{12}^{2} \frac{E_{2}}{E_{1}}}, \quad Q_{12}=\nu_{12} Q_{22}, \quad Q_{66}=G_{12}
$$




\section{References}

[1] C. R. Farrar, N. A. Lieven, Damage prognosis: the future of structural health monitoring, Philosophical Transactions of the Royal Society A: Mathematical, Physical and Engineering Sciences 365 (1851) (2007) 623-632.

[2] I. Lopez, N. Sarigul-Klijn, A review of uncertainty in flight vehicle structural damage monitoring, diagnosis and control: Challenges and opportunities, Progress in Aerospace Sciences 46 (7) (2010) 247-273.

[3] D. J. Inman, C. R. Farrar, V. L. Junior, V. S. Junior, Damage Prognosis: For Aerospace, Civil and Mechanical Systems, John Wiley \& Sons, 2005.

[4] Y. Ling, S. Mahadevan, Integration of structural health monitoring and fatigue damage prognosis, Mechanical Systems and Signal Processing 28 (2012) 89-104.

[5] K. Reifsnider, A. Talug, Analysis of fatigue damage in composite laminates, International Journal of Fatigue 2 (1) (1980) $3-11$.

[6] R. Jamison, K. Schulte, K. Reifsnider, W. Stinchcomb, Characterization and analysis of damage mechanisms in tensiontension fatigue of graphite/epoxy laminates, Effects of defects in composite materials, ASTM STP 836 (1984) 21-55.

[7] F.-K. Chang, J. F. Markmiller, J.-B. Ihn, K. Y. Cheng, A potential link from damage diagnostics to health prognostics of composites through built-in sensors, Journal of Vibration and Acoustics 129 (6) (2007) 718-729.

[8] M. Chiachío, J. Chiachío, G. Rus, Reliability in composites - A selective review and survey of current development, Composites Part B 43 (3) (2012) 902-913.

[9] S. Sankararaman, Significance, interpretation, and quantification of uncertainty in prognostics and remaining useful life prediction, Mechanical Systems and Signal Processing.

[10] M. E. Orchard, G. J. Vachtsevanos, A particle filtering-based framework for real-time fault diagnosis and failure prognosis in a turbine engine, in: Proceedings of the Mediterranean Conference on Control \& Automation, 2007, IEEE, 2007, pp. $1-6$.

[11] A. Heng, S. Zhang, A. C. Tan, J. Mathew, Rotating machinery prognostics : State of the art, challenges and opportunities, Mechanical Systems and Signal Processing 23 (2009) 724-739.

[12] M. Gašperin, Đ. Juričić, P. Boškoski, J. Vižintin, Model-based prognostics of gear health using stochastic dynamical models, Mechanical Systems and Signal Processing 25 (2) (2011) 537-548.

[13] M. Daigle, K. Goebel, A model-based prognostics approach applied to pneumatic valves, International Journal of the PHM Society 2 (8) (2010) 1:16.

[14] M. Daigle, K. Goebel, Model-based prognostics with fixed-lag particle filters, in: Proceedings of the Annual Conference of the Prognostics and Health Management Society, 2019, Vol. 1, 2009, pp. 249-261.

[15] E. Zio, G. Peloni, Particle filtering prognostic estimation of the remaining useful life of nonlinear components, Reliability Engineering \& System Safety 96 (3) (2011) 403-409.

[16] Y. Ling, C. Shantz, S. Mahadevan, S. Sankararaman, Stochastic prediction of fatigue loading using real-time monitoring data, International Journal of Fatigue 33 (7) (2011) 868-879.

[17] M. Gobbato, J. B. Kosmatka, J. P. Conte, A recursive Bayesian approach for fatigue damage prognosis: an experimental validation at the reliability component level, Mechanical Systems and Signal Processing 45 (2014) 448-467.

[18] J. Sikorska, M. Hodkiewicz, L. Ma, Prognostic modelling options for remaining useful life estimation by industry, Mechanical Systems and Signal Processing 25 (5) (2011) 1803-1836.

[19] M. Gobbato, J. P. Conte, J. B. Kosmatka, C. R. Farrar, A reliability-based framework for fatigue damage prognosis of composite aircraft structures, Probabilistic Engineering Mechanics 29 (2012) 176-188.

[20] J. Chiachío, M. Chiachío, A. Saxena, G. Rus, K. Goebel, An energy-based prognostics framework to predict fatigue damage evolution in composites, in: Proceedings of the Annual Conference of the Prognostics and Health Management Society, 2013, Vol. 1, Prognostics and Health Management Society, 2013, pp. 363-371. 
[21] M. Chiachío, J. Chiachío, A. Saxena, G. Rus, K. Goebel, Fatigue damage prognosis in FRP composites by combining multi-scale degradation fault modes in an uncertainty Bayesian framework, in: F.-K. Chang (Ed.), Proceedings of the 9th Structural Health Monitoring, 2013, Vol. 1, 2013, pp. 1368-1375.

[22] N. Gordon, D. Salmond, A. Smith, Novel approach to nonlinear/non-Gaussian Bayesian state estimation, IEEEProceedings-F 140 (1993) 107-113.

[23] M. Arumlampalam, S. Maskell, N. Gordon, T. Clapp, A tutorial on particle filters for on-line nonlinear/non-Gaussian Bayesian tracking, IEEE Transactions on Signal Processing 50 (2) (2002) 174-188.

[24] M. Orchard, G. Kacprzynski, K. Goebel, B. Saha, G. Vachtsevanos, Advances in uncertainty representation and management for particle filtering applied to prognostics, in: International conference on prognostics and health management, IEEE, 2008.

[25] E. Myötyri, U. Pulkkinen, K. Simola, Application of stochastic filtering for lifetime prediction, Reliability Engineering \& System Safety 91 (2) (2006) 200-208.

[26] M. E. Orchard, G. J. Vachtsevanos, A particle-filtering approach for on-line fault diagnosis and failure prognosis, Transactions of the Institute of Measurement and Control 31 (3-4) (2009) 221-246.

[27] B. Zhang, C. Sconyers, C. Byington, R. Patrick, M. Orchard, G. Vachtsevanos, A probabilistic fault detection approach: application to bearing fault detection, Industrial Electronics, IEEE Transactions on 58 (5) (2011) 2011-2018.

[28] P. Baraldi, F. Mangili, E. Zio, Investigation of uncertainty treatment capability of model-based and data-driven prognostic methods using simulated data, Reliability Engineering \& System Safety 112 (2013) 94-108.

[29] D. An, J.-H. Choi, N. H. Kim, Prognostics 101: A tutorial for particle filter-based prognostics algorithm using Matlab, Reliability Engineering \& System Safety 115 (2013) 161-169.

[30] J. Sun, H. Zuo, W. Wang, M. G. Pecht, Prognostics uncertainty reduction by fusing on-line monitoring data based on a state-space-based degradation model, Mechanical Systems and Signal Processing 45 (2) (2014) 396-407.

[31] E. Jaynes, Probability Theory: The Logic of Science, Cambridge University Press, 2003.

[32] J. L. Beck, Bayesian system identification based on probability logic, Structural Control and Health Monitoring 17 (2010) $825-847$.

[33] A. Saxena, K. Goebel, C. Larrosa, F. Chang, CFRP Composites dataset, NASA Ames Prognostics Data Repository, [http://ti.arc.nasa.gov/project/prognostic-data-repository], NASA Ames, Moffett Field, CA (2008).

[34] J. A. Nairn, in: R. Talreja, M. J.A.E. (Eds.), Polymer Matrix Composites, Elsevier Science, Amsterdam, 2000, Ch. Matrix Microcracking in Composites, pp. 403-432.

[35] R. Talreja, C. V. Singh, Damage and Failure of Composite Materials, Cambridge University Press, 2012.

[36] K. Garrett, J. Bailey, Multiple transverse fracture in $90^{\circ}$ cross-ply laminates of a glass fibre-reinforced polyester, Journal of Materials Science 12 (1) (1977) 157-168.

[37] A. Highsmith, K. Reifsnider, Stiffness-reduction mechanisms in composite laminates, Damage in composite materials, ASTM STP 775 (1982) 103-117.

[38] Z. Hashin, Analysis of cracked laminates: a variational approach, Mechanics of Materials 4 (2) (1985) 121-136.

[39] P. Gudmundson, Z. Weilin, An analytic model for thermoelastic properties of composite laminates containing transverse matrix cracks, International Journal of Solids and Structures 30 (23) (1993) 3211-3231.

[40] P. Lundmark, J. Varna, Constitutive relationships for laminates with ply cracks in in-plane loading, International Journal of Damage Mechanics 14 (3) (2005) 235-259.

[41] J. Chiachío, M. Chiachío, A. Saxena, S. Sankararaman, G. Rus, K. Goebel, Bayesian model class selection and parameter estimation for fatigue damage progression in composites, International Journal of Fatigue (2014) http://dx.doi.org/10.1016/j.ijfatigue.2014.08.003.

[42] P. W. Manders, T.-W. Chou, F. R. Jones, J. W. Rock, Statistical analysis of multiple fracture in 0/90/0 glass fibre/epoxy 
resin laminates, Journal of Materials Science 18 (10) (1983) 2876-2889.

[43] R. Joffe, J. Varna, Analytical modeling of stiffness reduction in symmetric and balanced laminates due to cracks in 90 layers, Composites Science and Technology 59 (11) (1999) 1641-1652.

[44] J. A. Nairn, The strain energy release rate of composite microcracking: a variational approach, Journal of Composite Materials 23 (11) (1989) 1106-1129.

[45] J. Nairn, S. Hu, The initiation and growth of delaminations induced by matrix microcracks in laminated composites, International Journal of Fracture 57 (1) (1992) 1-24.

[46] A. Doucet, N. De Freitas, N. Gordon, Sequential Monte Carlo methods in practice, Springer Verlag, 2001.

[47] E. T. Jaynes, Papers on Probability, Statistics and Statistical physics, (Ed. R.D. Rosenkrantz) Kluwer Academic Publishers, 1983.

[48] A. Papoulis, Probability, Random Variables, and Stochastic Processes, McGraw Hill, 1965.

[49] A. Saltelli, M. Ratto, S. Tarantola, F. Campolongo, Sensitivity analysis practices: Strategies for model-based inference, Reliability Engineering \& System Safety 91 (10) (2006) 1109-1125.

[50] J. Liu, M. West, Combined parameter and state estimation in simulation-based filtering, in: A. Doucet, N. Freitas, N. Gordon (Eds.), Sequential Monte Carlo Methods in Practice, Statistics for Engineering and Information Science, Springer New York, 2001, pp. 197-223.

[51] M. J. Daigle, K. Goebel, Model-based prognostics with concurrent damage progression processes, Systems, Man, and Cybernetics: Systems, IEEE Transactions on 43 (3) (2013) 535-546.

[52] H. Tanizaki, R. S. Mariano, Nonlinear and non-Gaussian state-space modeling with Monte Carlo simulations, Journal of Econometrics 83 (1) (1998) 263-290.

[53] A. Kong, J. S. Liu, W. H. Wong, Sequential imputations and bayesian missing data problems, Journal of the American statistical association 89 (425) (1994) 278-288.

[54] H. Lu, W. J. Kolarik, S. S. Lu, Real-time performance reliability prediction, IEEE Transactions on Reliability 50 (4) (2001) 353-357.

[55] Z. Zhao, Q. Quan, K.-Y. Cai, A profust reliability based approach to prognostics and health management, IEEE Transactions on Reliability 63 (1) (2014) 26-41.

[56] C. Larrosa, F. Chang, Real time in-situ damage classification, quantification and diagnosis for composite structures, in: Proceedings of the 19th International Congress on Sound and Vibration, Vol. 15, 2012.

[57] A. Saxena, K. Goebel, C. Larrosa, V. Janapati, S. Roy, F.-K. Chang, Accelerated aging experiments for prognostics of damage growth in composites materials, in: The 8th International Workshop on Structural Health Monitoring, F.K. Chang, Editor., Vol. 15, 2011.

[58] A. Saxena, J. Celaya, B. Saha, B. Saha, K. Goebel, Metrics for offline evaluation of prognostic performance, International Journal of the PHM Society 1 (1) (2010) 20.

[59] S. Tsai, Theory of composites design, Think composites, 1992.

[60] J. Reddy, Mechanics of Laminated Composite Plates and Shells: Theory and Analysis, Second Edition, CRC Mechanical Engineering Series. 


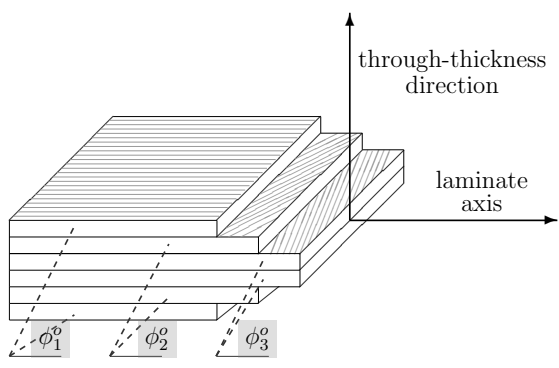

(a)

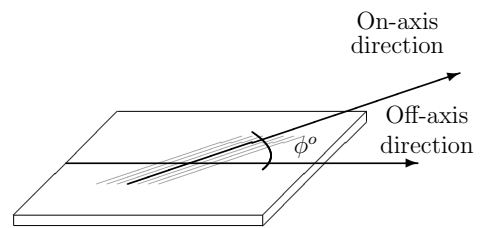

(b)

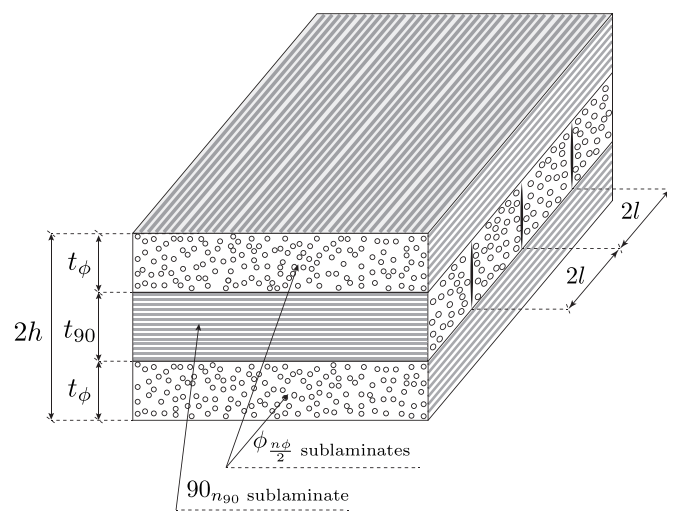

(c)

Figure A.1: Panel a): Schematic view of a FRP composite laminate with stacking sequence given by $\left[\phi_{1}^{o} / \phi_{2}^{o} / \phi_{3}^{o}\right]_{S}$. Panel b): Illustration of one of the plies with indication of ply and laminate directions. Panel c): Illustration of microscopic damage for a $\left[\phi_{\frac{n_{\phi}}{2}} / 90_{n_{90}} / \phi_{\frac{n_{\phi}}{2}}\right]$ laminate (as the laminate used in the case study) along with basic geometrical parameters.

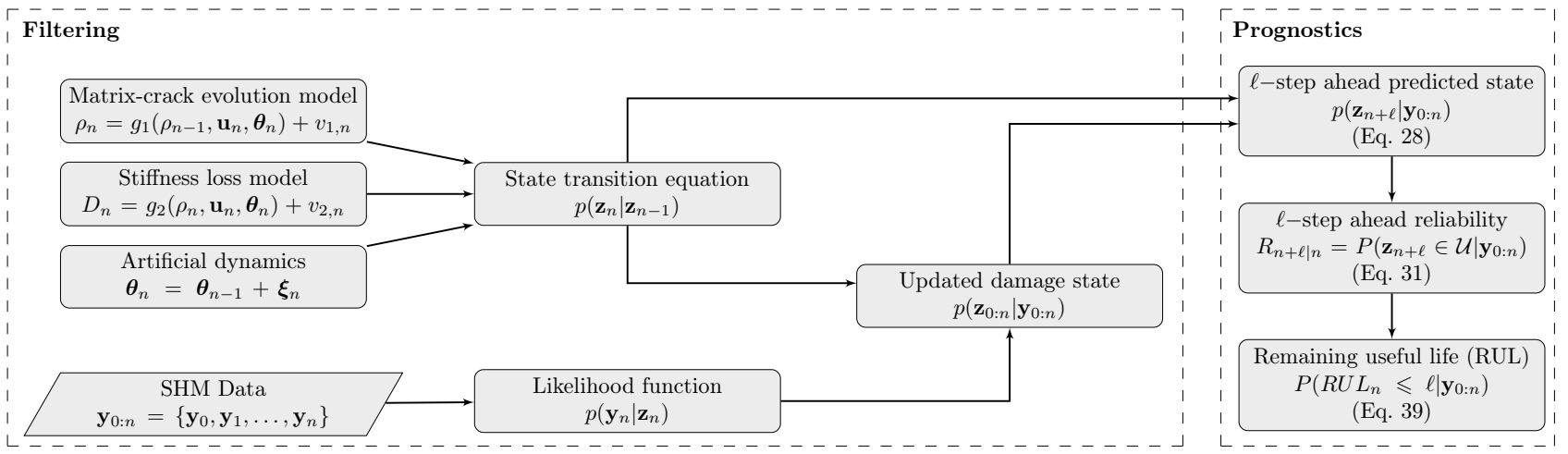

Figure A.2: Conceptual scheme for prognostics based on time-dependent reliability. Every time new data are collected, the damage state is updated and further propagated forward in time whereby the time-dependent reliability is predicted. As by-product, a estimation of RUL is obtained. 

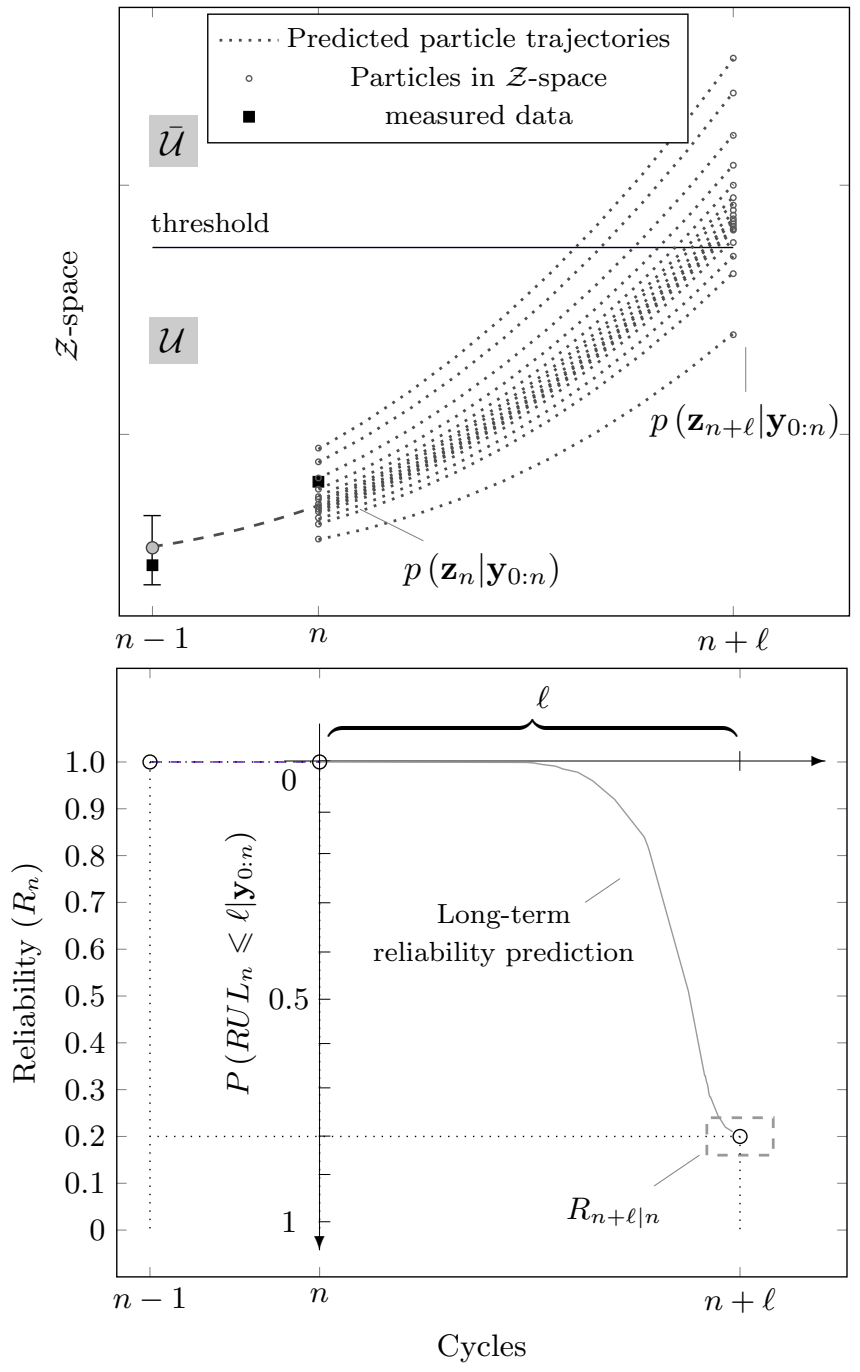

Figure A.3: Illustrative example of the proposed framework for prognostics based on time-dependent reliability. Top panel: samples of $\mathbf{z}$-states along with their idealized sample trajectories against time steps $\{n-1, n, \ldots, n+\ell\}$, where $n$ is the last time when data become available. The horizontal line represents the boundary between the useful domain $\mathcal{U}$ and its complementary region $\overline{\mathcal{U}}$. Bottom panel: predicted reliability from time $n$. Observe the correspondence between the predicted reliability $R_{n+\ell \mid n}$ and the $P\left(R U L_{n} \leqslant \ell \mid \mathbf{y}_{0: n}\right)$, as shown in Eq. 39. 


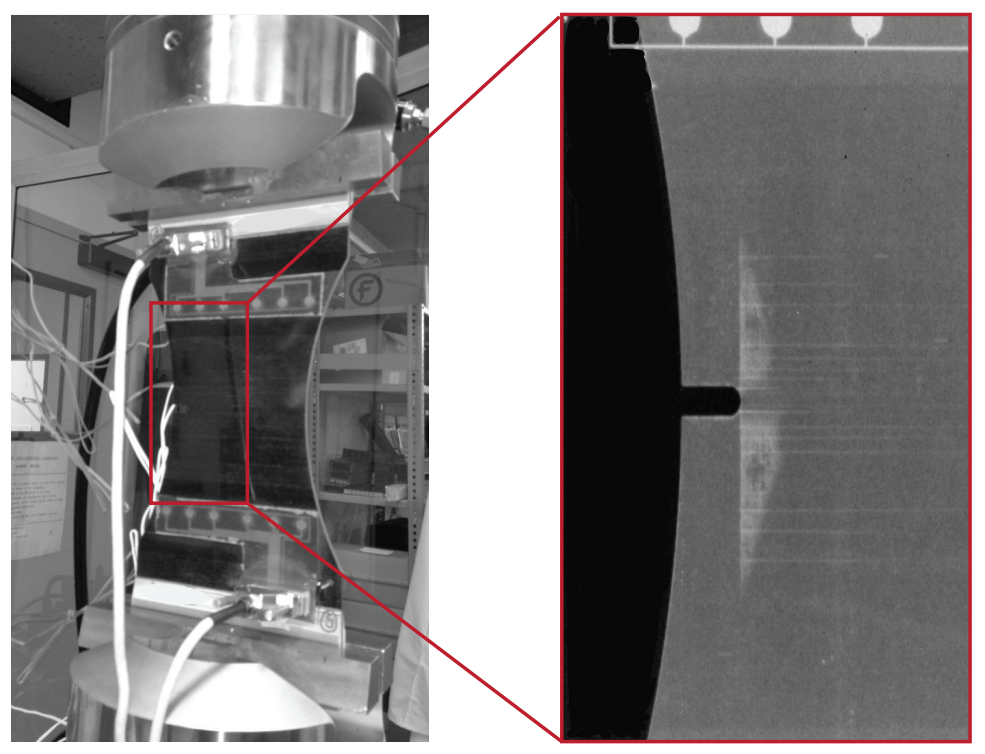

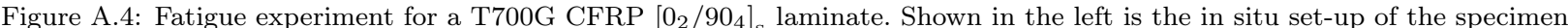
on the testing machine. Observe the SHM system based on PZT sensors (SMART Layer $\AA$ from Acellent Technologies Inc), which are placed on top and bottom of the specimen. The right panel shows a X-ray image of the specimen after 100 fatigue cycles. The bright white areas denote delaminated interfaces whereas the horizontal white lines are matrix cracks. 


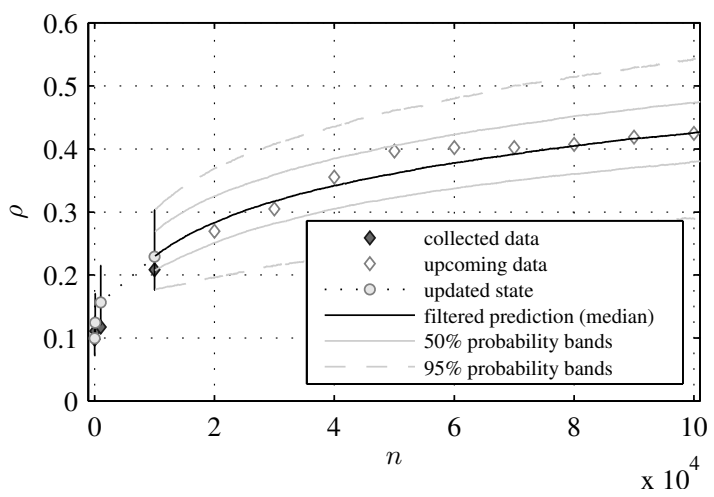

(a)

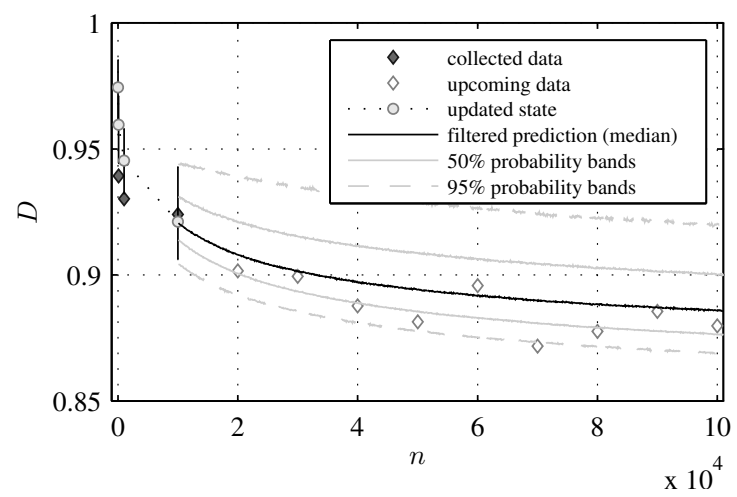

(b)

$\left(n=1 \cdot 10^{4}\right)$

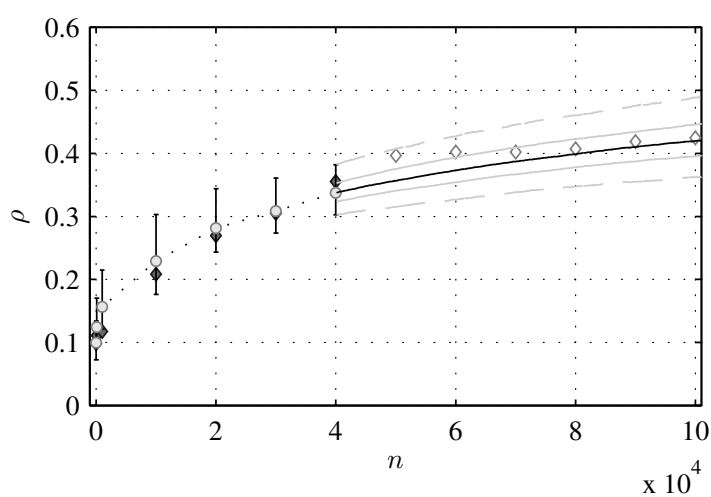

(c)

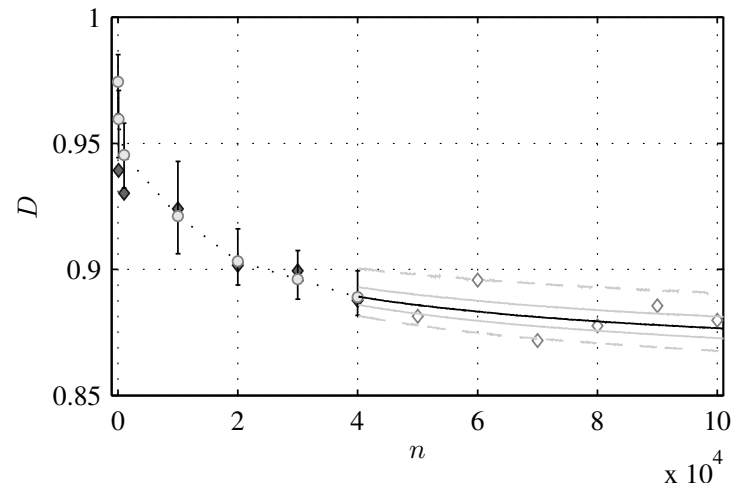

(d)

$\left(n=4 \cdot 10^{4}\right)$

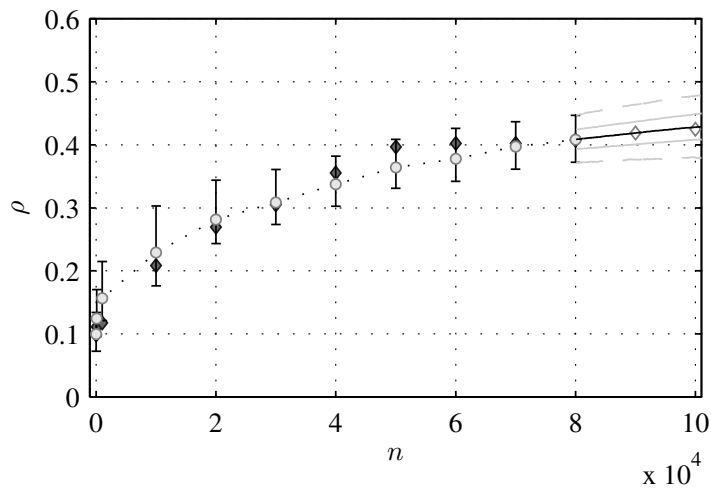

(e)

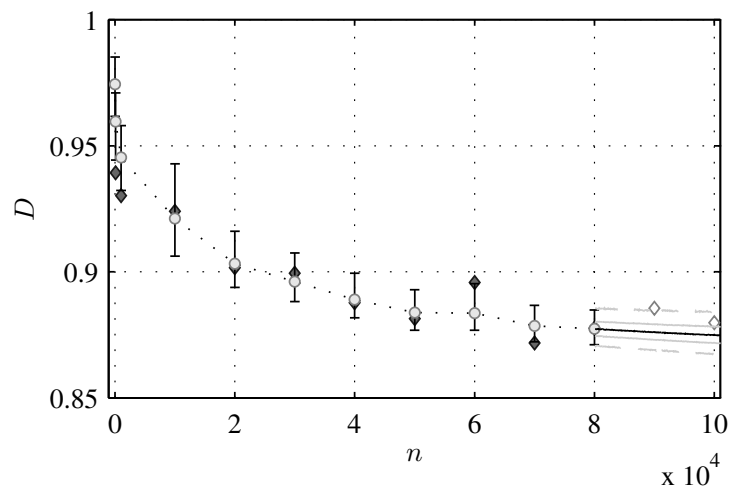

$(\mathrm{f})$

$$
\left(n=8 \cdot 10^{4}\right)
$$

Figure A.5: Sequential state estimation for matrix micro-cracks density (\# cracks $/ \mathrm{mm}$ ) and normalized effective stiffness (dimensionless) up to a certain cycle $n$, where $n=1 \times 10^{4}$ (a \& b), $4 \times 10^{4}$ (c \& d) and $8 \times 10^{4}$ (e \& f). The multi-step ahead predicted damage states are represented using dashed gray lines for the $5 \%-95 \%$ probability bands and solid gray lines for the $25 \%-75 \%$ probability bands. 


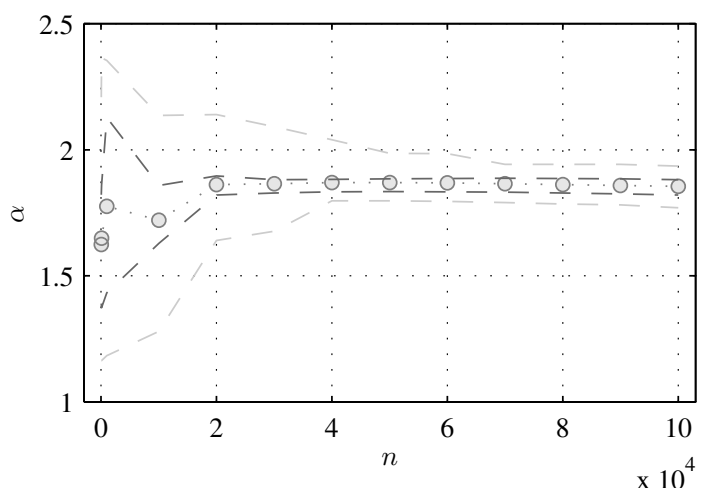

(a) $\theta_{1}$

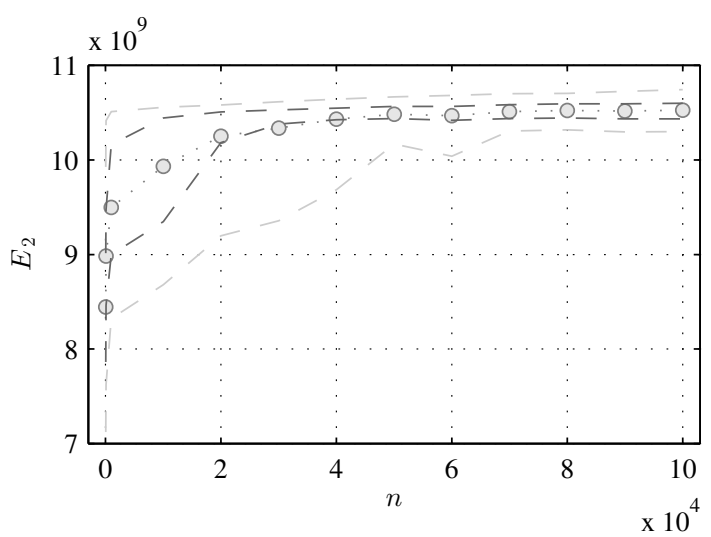

(c) $\theta_{3}$

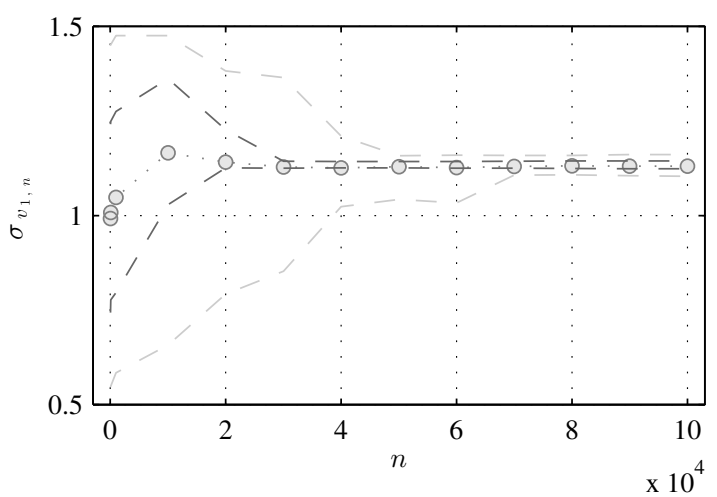

(e) $\theta_{5}$

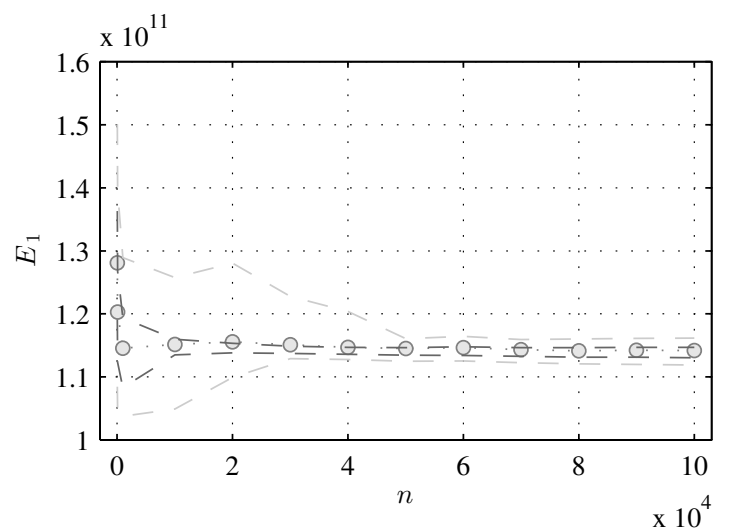

(b) $\theta_{2}$

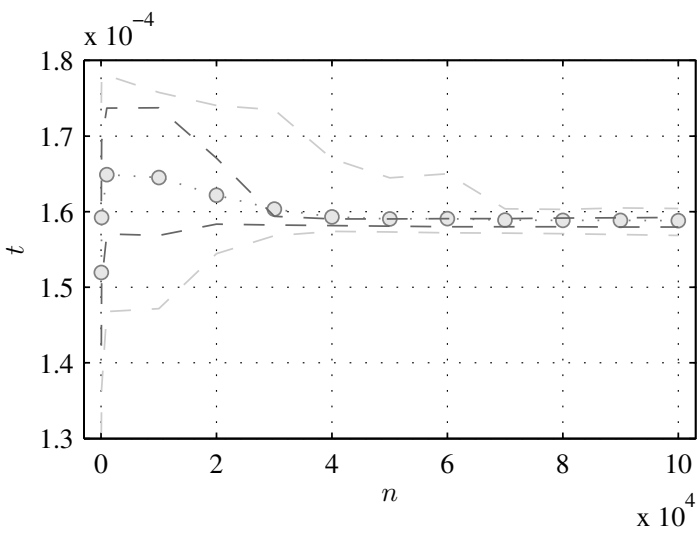

(d) $\theta_{4}$

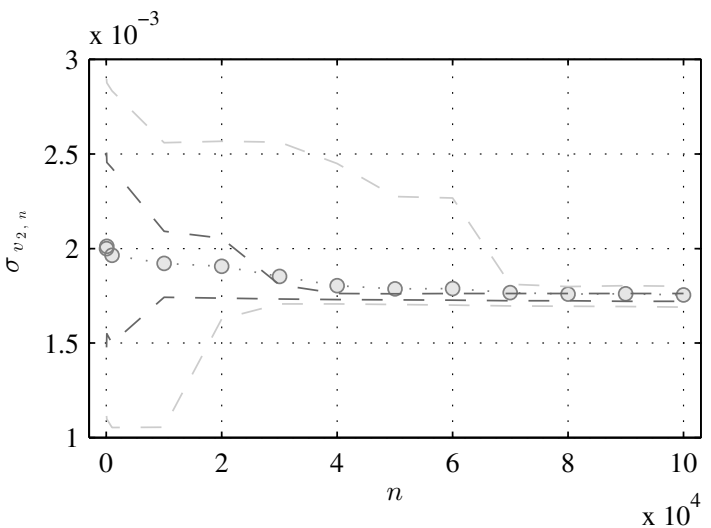

(f) $\theta_{6}$

Figure A.6: Trace of the mean values of model parameters $\boldsymbol{\theta}$ against cycles. Dashed lines represent the $25 \%-75 \%$ (darker color) and 5\% - 95\% probability bands, respectively. 


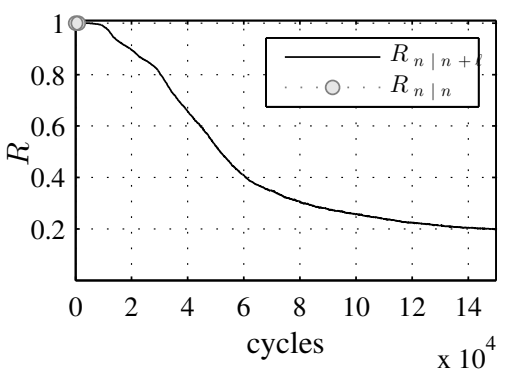

(a)

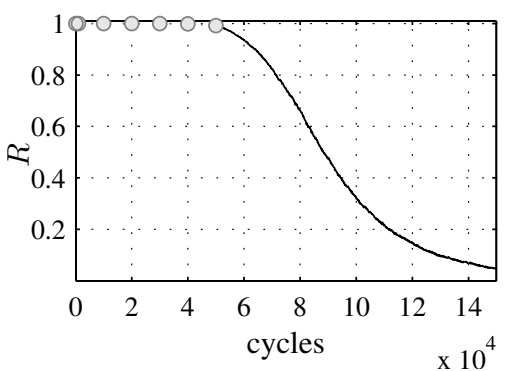

(d)

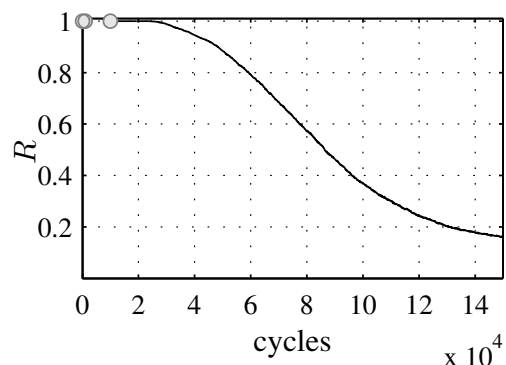

(b)

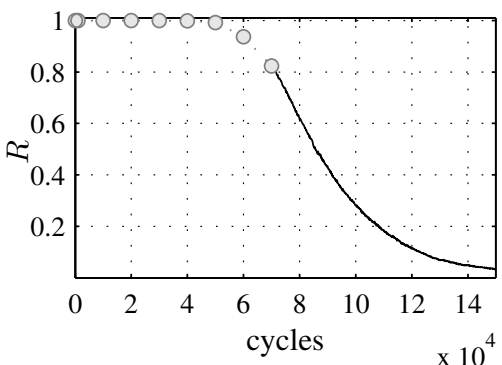

(e)

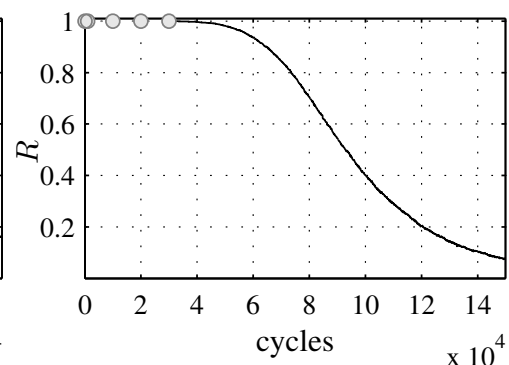

(c)

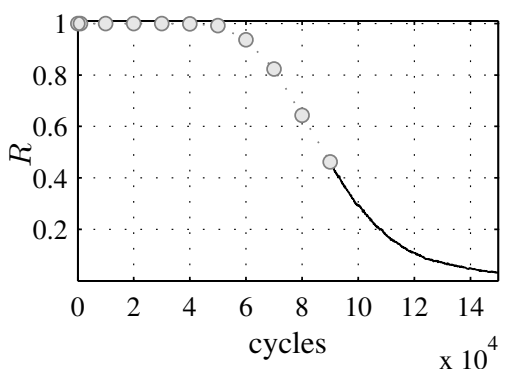

(f)

Figure A.7: Plot of reliability updating and time-dependent prediction at selected cycles $n$, where $n=\{0.1,1,3,5,7,9\} \times 10^{4}$ for panels (a) to (f), respectively. The gray circles represent the updated reliability values up to cycle $n$, whereas the solid curves correspond to the reliability prediction for upcoming fatigue cycles.

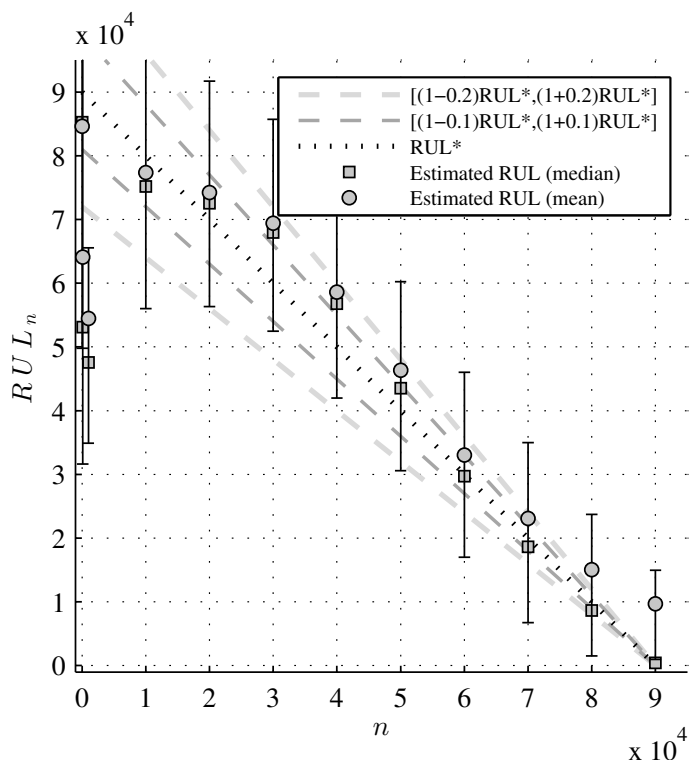

Figure A.8: RUL estimation vs. fatigue cycles obtained from the proposed reliability based prognostics approach. Observe that the uncertainty in RUL prediction (represented by interquartile bars) decreases as long as new data become available. 


\begin{tabular}{lccccccccccccc}
\hline Fatigue cycles, $n$ & $10^{1}$ & $10^{2}$ & $10^{3}$ & $10^{4}$ & $2 \cdot 10^{4}$ & $3 \cdot 10^{4}$ & $4 \cdot 10^{4}$ & $5 \cdot 10^{4}$ & $6 \cdot 10^{4}$ & $7 \cdot 10^{4}$ & $8 \cdot 10^{4}$ & $9 \cdot 10^{4}$ & $10^{5}$ \\
\hline$\rho_{n}[\#$ cracks $/ m]$ & 98.2 & 111.0 & 117.4 & 208.5 & 269.6 & 305.0 & 355.5 & 396.4 & 402.3 & 402.1 & 407.0 & 418.5 & 424.5 \\
$D_{n}$ & 0.954 & 0.939 & 0.930 & 0.924 & 0.902 & 0.899 & 0.888 & 0.881 & 0.896 & 0.872 & 0.877 & 0.885 & 0.880 \\
\hline
\end{tabular}

Table A.1: Experimental sequence of damage for the cross-ply $\left[0_{2} / 90_{4}\right]_{\mathrm{S}}$ Torayca T700 CFRP laminate used in the case study. The data are presented for micro-cracks density $\left(\rho_{n}\right)$ and normalized effective stiffness $\left(D_{n}\right)$.

\begin{tabular}{lrrcr}
\hline Type & Parameter & Nominal value & Units & Prior PDF \\
\hline Mechanical & $E_{1}\left(\theta_{1}\right)$ & $127.55 \cdot 10^{9}$ & $\mathrm{~Pa}$ & $\mathcal{L N}\left(\ln \left(127.55 \cdot 10^{9}\right), 0.1\right)$ \\
& $E_{2}\left(\theta_{2}\right)$ & $8.41 \cdot 10^{9}$ & $\mathrm{~Pa}$ & $\mathcal{L N}\left(\ln \left(8.41 \cdot 10^{9}\right), 0.1\right)$ \\
& $t\left(\theta_{3}\right)$ & $1.5 \cdot 10^{-4}$ & $m$ & $\mathcal{L} \mathcal{N}\left(\ln \left(1.5 \cdot 10^{-4}\right), 0.1\right)$ \\
& $G_{12}$ & $6.20 \cdot 10^{9}$ & $\mathrm{~Pa}$ & Not applicable \\
& $\nu_{12}$ & 0.31 & - & Not applicable \\
& $G_{23}$ & $2.82 \cdot 10^{9}$ & $\mathrm{~Pa}$ & Not applicable \\
\hline Fitting & $\alpha\left(\theta_{4}\right)$ & 1.80 & - & $\mathcal{L N}(\ln (1.80), 0.2)$ \\
& $A$ & $1 \cdot 10^{-4}$ & - & Not applicable \\
\hline Errors & $\sigma_{v_{1}}\left(\theta_{5}\right)$ & - & $\frac{\# \text { cracks }}{m \cdot c y c l e}$ & $\mathcal{U}(0.5,8)$ \\
& $\sigma_{v_{2}}\left(\theta_{6}\right)$ & - & - & $\mathcal{U}(0.001,0.02)$ \\
\hline
\end{tabular}

Table A.2: Nominal values and prior uncertainty of model parameters used in calculations. The rest of parameters in damage mechanics models (Eq. 1 to 6) are obtained using the classical laminate theory [60] and the relations given in Appendix A. The nominal values for fitting parameters have been defined through initial fitting tests.

\begin{tabular}{rrlcr}
\hline Laminate & & & & \\
& $E_{x}$ & Longitudinal Young's modulus & $t_{\phi}$ & {$\left[\phi_{\frac{n_{\phi}}{2}}\right]$-sublaminate thickness } \\
& $E_{x}^{*}$ & Effective long. Young's modulus & $t$ & Ply thickness \\
Sublaminate & & Laminate half-thickness & Ply & \\
& $E_{x}^{(\phi)}$ & Longitudinal Young's modulus & $E_{1}$ & Longitudinal Young's modulus \\
& $E_{y}^{(\phi)}$ & Transverse Young's modulus & $E_{2}$ & Transverse Young's modulus \\
$\nu_{x y}^{(\phi)}$ & In-plane Poisson ratio & $\nu_{12}$ & In-plane Poisson ratio \\
$G_{x y}^{(\phi)}$ & In-plane shear modulus & $\nu_{23}$ & Out-of-plane Poisson ratio \\
$G_{x z}^{(\phi)}$ & Out-of-plane shear modulus & $G_{12}$ & In-plane shear modulus \\
$t_{90}$ & {$\left[90_{n_{90}}\right]$-sublaminate half-thickness } & $G_{23}$ & Out-of-plane shear modulus \\
\hline
\end{tabular}

Table A.3: Nomenclature table. Nominal values of main ply and geometry parameters are provided in Table A.2. 